\title{
A COSMOLOGICAL HYDRODYNAMIC CODE BASED ON THE TOTAL VARIATION DIMINISHING SCHEME
}

\author{
Dongsu Ryu, Jeremiah P. Ostriker, Hyesung Kang, and Renyue Cen \\ Princeton University Observatory, Peyton Hall, Princeton, NJ 08544 \\ Received 1992 October 30; accepted 1993 March 5
}

\begin{abstract}
We describe an explicit second-order finite difference code based on a total variation diminishing scheme for self-gravitating cosmological hydrodynamic systems. The code has been developed to follow correctly the adiabatic changes of extremely supersonic preshock flows with a Mach number larger than 100 as well as very strong shocks. In highly supersonic regions, we use an entropy-like variable switching to a more conventional total energy variable near to and interior to shocks. The self-gravity has been included in such a way that the numerical errors in calculating the gravitational force term do not induce the leakage of the gravitational energy into the thermal energy of the gas. Also, the gravitational force term has been corrected to take account of the mass diffusion around the shocks so the total energy can be conserved. Tests for the accuracy and performance of the code without gravity have proved that it can accurately handle supersonic flows with a Mach number larger than $10^{4}$. In calculations of the formation of a one-dimensional Zel'dovich pancake, an energy accuracy of $1 \%$ is obtained for 32 cells per unit wavelength, and the accuracy reaches $0.01 \%$ as the number of cells approaching 1024. To further test the code with gravity, three-dimensional simulations of a purely baryonic universe but with the initial cold dark matter power-spectrum have been performed. The results have shown that shocks are well resolved and separate cleanly the hot, dense, collapsed peaks from the cold, low-density, expanding voids. The thermal energy in low-density regions can be orders of magnitude lower using this scheme than in some others due to very careful attention given to entropy in high Mach number regions. Various numerical experiments have proven that the code can handle the expanding low density regions very well as well as conserve the total energy very accurately.
\end{abstract}

Subject headings: cosmology: theory - hydrodynamics - methods: numerical - shock waves

\section{INTRODUCTION}

The universe is considered to be composed of collisionless dark matter and collisional baryonic matter in commonly adopted models. The total mass density of the universe can be estimated, for instance, by the dynamics of galaxies and, in units of the critical density, $\Omega \gtrsim 0.2$ (Peebles 1986). On the other hand, the baryonic matter density is limited by the theory of primordial nucleosynthesis and its upper bound is placed to be $\Omega_{\mathrm{BM}} \lesssim 0.1$ (Kurki-Suonio et al. 1990; Walker et al. 1991). Even though the dark matter seems to comprise the majority of the mass, we observe mostly the distribution of the baryonic matter, i.e., galaxies and clusters, and the signal from the baryonic matter, i.e., the $\mathrm{X}$-ray background radiation (Giacconi et al. 1962) and the distortion of the cosmic background radiation (CBR) spectrum due to the Zel'dovichSunyaev effect (Zel'dovich \& Sunyaev 1969; Ostriker \& Vishniac 1986).

Until recently, most studies on the evolution of the universe and the distribution of matter have used only the dark matter primarily due to the practical difficulties of incorporating hydrodynamics into cosmological simulations (see, e.g., Efstathiou et al. 1985; Centrella et al. 1988; Park 1990; Bertschinger \& Gelb 1991 and references therein for the studies on the dark matter universe with the $N$-body simulations). The available computers simply did not have enough power to treat simultaneously small scales like $l \lesssim 0.5 \mathrm{Mpc}$, at which the hydrodynamic effects become important, and large scales $l \gtrsim 50 \mathrm{Mpc}$, at which the effects of the nonlinearity of the structure provide the major entropy input. However, as the available computing resources have allowed us to include the gaseous components of the matter into the cosmological simulations with a physically meaningful resolution, more serious attempts to study the hydrodynamical aspects of the large-scale structure of the universe have been made (see, e.g., Chiang, Ryu, \& Vishniac 1989; Ryu, Vishniac, \& Chiang 1990; Cen et al. 1990; Cen et al. 1991; Cen 1992; Cen \& Ostriker 1992a, b, c; Yuan, Centrella, \& Norman 1991; Katz, Hernquist, \& Weinberg 1992).

The cosmological hydrodynamic simulations are much more challenging than the typical hydrodynamic simulations without self-gravity due to the following reasons:

1. The nonlinear structures grow due to the gravitational collapse, and subsequently extremely supersonic motion is generated around the density peaks while the background gas stays cold. In the extremely supersonic flow with a large Mach number, e.g., $M \gtrsim 100$, the thermal energy is very small compared to the kinetic energy $\left(E_{\mathrm{th}} \sim E_{\mathrm{kin}} / M^{2}\right)$. Hence, in the conventional hydrodynamic codes in which the total energy (the kinetic plus thermal energy) is used as a variable, the thermal energy cannot be tracked accurately even though the equation is of a conservative form. As a result, a significant amount of entropy could be generated, even before the flow becomes shocked.

2. The gravitational energy, which has a same order of magnitude as the kinetic energy, is also much larger than the thermal energy. Hence, a small leakage of the gravitational energy into the thermal energy due to numerical error could heat the background gas significantly.

3. The conventional codes add a finite amount of diffusion around shocks to handle them without generating oscillations. As a result, when the self-gravity is included, the total energy 
(the kinetic, thermal, plus gravitational energy) is not conserved.

4. Very complicated structures form on small scales, yet the resolution is very limited.

A good cosmological hydrodynamic code should be able to handle the above difficulties. The fourth difficulty requires a code which follows the structures accurately and reproduces the sharp gradients, and yet which is fast and uses a minimum memory. We built the code around the total variation diminishing (TVD) scheme, originally developed by Harten (1983). We overcome the first difficulty by solving the equation involving the thermal energy instead of the total energy equation in the region free of shocks, but still keeping the original accuracy of the TVD scheme. The second difficulty was bypassed by keeping the thermal and kinetic energies separately in the step to include the gravity and adding the effect of the gravity only to the kinetic energy. Finally, we overcome the third difficulty by adding the effect of mass diffusion to the gravitational force term around the shocks.

In the present work, we assume that the gas is adiabatic and the code comprises only the routines to handle the dynamics and gravity. The radiative processes and the ionization and recombination processes will be included in the future work. In $\S 2$, we present the basic description of the TVD scheme as well as its modification to handle the extremely supersonic flow. In $\S 3$, we expand the method to include the self-gravity and the cosmological terms in the cosmological hydrodynamic TVD code. In $\S 4$, we demonstrate the suitability of the code for the cosmological hydrodynamic simulations by presenting the results of several test numerical experiments. Finally, a brief summary is given in $\S 5$. In the following appendices, the details of the original TVD scheme and its modifications are described so that the readers can reconstruct the code without consulting other references.

\section{HYDRODYNAMIC CODE}

Cosmic plasma is not an ideal gas. Shocks are ubiquitous in astrophysical environments. In fact, most astrophysically interesting phenomena involve some kinds of supersonic motions. The kinetic energy of such supersonic flow is thermalized via molecular and/or electromagnetic viscosity inside the collisionless shocks. But, most often, the thickness of these shocks is very small compared to the length scale of our interests, so that they can be treated as discontinuous jumps in the flow. In the numerical hydrodynamics as well as in the theoretical studies, we can model the cosmic gas as an ideal compressible fluid instead of a viscous fluid as long as the conservation equations are solved correctly. In numerical hydrodynamic codes, however, numerical "dissipation" has to be adopted in order to handle the discontinuities (e.g., shocks and contact discontinuities). The quality of a hydrodynamic code is often measured by how well it can capture the discontinuities without generating nonphysical oscillations.

\subsection{Hydrodynamic Equations}

We solve the hyperbolic system of the following conservation equations for an ideal gas:

$$
\begin{gathered}
\frac{\partial \rho}{\partial t}+\frac{\partial}{\partial x_{k}}\left(\rho u_{k}\right)=0, \\
\frac{\partial\left(\rho u_{i}\right)}{\partial t}+\frac{\partial}{\partial x_{k}}\left(\rho u_{i} u_{k}+p \delta_{i k}\right)=0,
\end{gathered}
$$

$$
\frac{\partial E}{\partial t}+\frac{\partial}{\partial x_{k}}\left[(E+p) u_{k}\right]=0,
$$

where $E=p /(\gamma-1)+\rho u_{k}^{2} / 2$ is the total energy per unit volume and the rest of the variables have their usual meanings. In the conventional numerical schemes in which the mass, momentum, and total energy conservation equations are solved, the thermal energy is calculated by subtracting the kinetic energy from the total energy $E$.

For our new scheme, we introduce another conservative quantity, $S \equiv p / \rho^{\gamma-1}$, the modified entropy. By combining the internal energy equation with the mass conservation equation in (2.1), one can easily show that the modified entropy satisfies the following conservation equation:

$$
\frac{\partial S}{\partial t}+\frac{\partial}{\partial x_{k}}\left(S u_{k}\right)=0
$$

One should note that, unlike the internal energy equation, equation (2.4) has a conservative form. With equations (2.1) and (2.2), it forms another hyperbolic system of the conservative equations, which suggests that one can build an upwind scheme with them. Of course, this modified entropy equation is valid only outside the shock where the entropy is conserved.

It is interesting to note parenthetically that one can introduce an infinite number of conservative quantities,

$$
S_{\alpha}=p^{\alpha} \rho^{1-\gamma \alpha} \quad(-\infty<\alpha<\infty)
$$

which satisfy the conservation equation like that in equation (2.4). For $\alpha=0$, it reduces into the density and, for $\alpha=1$, into the modified entropy. Another interesting and useful choice would be $\alpha=1 / \gamma$, for which $S_{\alpha}$ reduces to $p^{1 / \gamma}$.

\subsection{TVD-E Code}

Harten's TVD scheme (Harten 1983) is an explicit, secondorder, Eulerian finite difference scheme which solves a hyperbolic system of the conservation equations. It is a nonlinear scheme obtained by first modifying the flux function and then applying a nonoscillatory first-order accurate scheme to get a resulting second-order accuracy. The key merit of this scheme is to achieve the high resolution of a second-order accuracy while preserving the robustness of the nonoscillatory first order scheme. Also, the scheme is relatively simple to program compared to other high-accuracy numerical schemes and requires less CPU time.

Harten (1983) described the application of the TVD scheme to the set of one-dimensional hydrodynamic equations and the test results. The multidimensional version of the TVD code is obtained by a Strang-type dimensional splitting (Strang 1968). While it is straightforward to expand the one-dimensional TVD code into the three-dimensional TVD code, the detailed implementation is somewhat involved. In Appendix A, we present the definition of some terminology used in the TVD scheme and reproduce the eigenvalues and eigenvectors for the three-dimensional hydrodynamic equations. And in Appendix $B$, we describe briefly the procedure to implement the TVD scheme to the system of the hydrodynamic equations. From now on, we refer the three-dimensional TVD code which solves equations (2.1)-(2.3) as the TVD-E code.

Our numerical tests (see, e.g., $\S 4$ ) showed that the TVD code gives the accuracy somewhat comparable in some cases to and somewhat worse in other cases than the high accuracy PPM (piecewise parabolic method: Colella \& Woodward 1984) 
code. However, the one-dimensional TVD code is approximately 5-6 times faster than the one-dimensional PPM code, and the three-dimensional TVD code is approximately 2-3 times faster.

\subsection{TVD-ES Code}

Inside the shock and in the postshock region where the thermal energy is necessarily comparable to the kinetic energy, the original TVD-E code solving equations (2.1)-(2.3) is adequate. However, suppose we are interested in the flow in which not only strong shocks are present but also the flow itself is highly supersonic, that is, the flow is in a gas which is very cold compared to the bulk kinetic energy (e.g., $E_{\text {th }} \lesssim 10^{-4} E_{\text {kin }}$, where $E_{\mathrm{th}}$ is the thermal energy and $E_{\text {kin }}$ is the kinetic energy) in the preshock region. This type of the flow cannot be treated properly with the original TVD-E code, because the numerical errors in calculating the total energy, although small, are much larger than the thermal energy itself.

We have overcome this difficulty by solving the modified entropy equation in equation (2.4) instead of the total energy equation and calculating the thermal energy directly in the region with $E_{\text {th }} / E_{\text {kin }} \ll 1$, that is, in the preshock region. Unlike the thermal energy equation or the pressure equation, the modified entropy equation has a conservative form. It forms a hyperbolic set of conservation equations with equations (2.1) and (2.2). So we can apply the TVD scheme to the new set of equations and obtain a code with similar accuracy to the original TVD-E code but now the variable is directly related to internal energy. In Appendix $\mathrm{C}$, we have derived the eigenvalues and eigenvectors for the new set of equations which are used to build the TVD-S code.

Now, let us explain the ES approach. We update the hydrodynamic quantities with two hyperbolic systems, one for equations (2.1)-(2.3) (E system) and one for equations (2.1), (2.2) and (2.4) (S system). While we choose the density and momentum which are updated with the $\mathrm{E}$ system, the thermal energy is chosen from the results of either the $\mathrm{E}$ system or the $\mathrm{S}$ system depending on the flow characteristics. The thermal energy updated with the $\mathrm{S}$ system is valid only in the regions without shocks, since the modified entropy equation is valid only outside shocks. On the other hand, the thermal energy updated with the $E$ system is accurate inside the shocks and in the regions where the flow is not extremely supersonic. After various numerical experiments, we have adopted the following criteria both of which must be satisfied for choosing when to implement the S system:

1. The flow is very supersonic.

$$
\frac{E_{\mathrm{th}}}{E}<0.02
$$

2. The flow is not undergoing a shock,

$$
u_{k}(j+1)-u_{k}(j-1) \geq 0,
$$

or

$$
\frac{\mid p(j+1)-p(j-1)}{p(j)} \leq 0.3 .
$$

In the above criteria, $E_{\mathrm{th}}$ and $p$ from the $S$ system and $E$ and $u_{k}$ from the $E$ system are used. Our numerical tests showed that, by efficiently combining the part to solve the $\mathrm{E}$ system with that for the $\mathrm{S}$ solver, the computational cost increases by about
$30 \%$. We refer the code described in this section as the TVD-ES code.

\section{COSMOLOGICAL HYDRODYNAMIC CODE}

\subsection{Cosmological Equations}

In comoving coordinates, the cosmological hydrodynamic equations with self-gravity and the modified entropy equation become

$$
\begin{gathered}
\frac{\partial \rho}{\partial t}+\frac{1}{a} \frac{\partial}{\partial x_{k}}\left(\rho u_{k}\right)=0 \\
\frac{\partial\left(\rho u_{i}\right)}{\partial t}+\frac{1}{a} \frac{\partial}{\partial x_{k}}\left(\rho u_{i} u_{k}+p \delta_{i k}\right)=-\frac{\dot{a}}{a} \rho u_{i}-\frac{1}{a} \rho \frac{\partial \phi}{\partial x_{i}} \\
\frac{\partial E}{\partial t}+\frac{1}{a} \frac{\partial}{\partial x_{k}}\left[(E+p) u_{k}\right]=-\frac{2 \dot{a}}{a} E-\frac{1}{a} \rho \mu_{k} \frac{\partial \phi}{\partial x_{k}} \\
\frac{\partial S}{\partial t}+\frac{1}{a} \frac{\partial}{\partial x_{k}}\left(S u_{k}\right)=-2 \frac{\dot{a}}{a} S
\end{gathered}
$$

where we have implicitly assumed $\gamma=5 / 3$. Here, $\rho$ is the comoving density, $u_{i}$ is the proper peculiar velocity, $p$ is the comoving pressure $\left[(\gamma-1) E_{\mathrm{th}}\right], E$ is the peculiar energy per unit comoving volume, $S$ is the comoving modified entropy, $\phi$ is the proper peculiar gravitational potential, $t$ is the cosmic time, and $a$ is the expansion parameter. The equation of state for the energy equation is $E \equiv p /(\gamma-1)+\rho u_{k}^{2} / 2$, and that for the modified entropy equations is $S \equiv p / \rho^{\gamma-1}$.

Similarly, the equations for the collisionless dark matter in comoving coordinates are written as

$$
\begin{gathered}
\frac{d x_{i}}{d t}=\frac{1}{a} u_{i}, \\
\frac{d u_{i}}{d t}=-\frac{\dot{a}}{a} u_{i}-\frac{1}{a} \frac{\partial \phi}{\partial x_{i}},
\end{gathered}
$$

where $x_{i}$ is the comoving coordinates. The proper peculiar gravitational potential is given by the Poisson equation

$$
\nabla^{2} \phi=\frac{4 \pi G}{a}\left(\rho_{\text {tot }}-\rho_{0}\right)
$$

where $\rho_{\text {tot }}$ is the total (baryonic and dark matter) comoving density and $\rho_{0}$ is the comoving background density.

In the code, we have normalized the variables with (1) the comoving length unit of the computational box size, $L_{\text {box }},(2)$ the time unit of the free fall time of the initial background density, $t_{G}=1 /\left(4 \pi G \rho_{i}\right)^{1 / 2}$, and (3) the density unit of the initial background density, $\rho_{i}$. Then, other variables in the equations are normalized as follows: $u_{i} /\left(L_{\text {box }} / t_{G}\right), \quad p /\left(\rho_{i} L_{\text {box }}^{2} / t_{G}^{2}\right)$, $E /\left(\rho_{i} L_{\text {box }}^{2} / t_{G}^{2}\right), S /\left(\rho_{i}^{2-\gamma} L_{\text {box }}^{2} / t_{G}^{2}\right)$, and $\phi /\left(L_{\text {box }}^{2} / t_{G}^{2}\right)$. We set the initial expansion parameter to be $a_{i}=1$, and the initial time is related to the redshift as $t_{i}=(2 / 3)\left(1 / H_{0}\right)\left(1+z_{i}\right)^{-3 / 2}$ in an $\Omega=1$ universe where $H_{0}$ is the present value of the Hubble parameter.

\subsection{Cosmological TVD-ES Code}

The details of each step to build our cosmological TVD-ES code are described schematically in Appendix D. Here is a brief summary. We consider the flux terms on the left-hand side of equations (3.1)-(3.4) as the hydrodynamic terms and the gravitational terms and the adiabatic expansion terms in the righthand side as the source terms. The hydrodynamic terms are 
taken account of in the hydrodynamic step, while the source terms in the gravitational and expansion steps. Before the hydrodynamic step, we update the velocity and pressure of the - baryonic matter by including the gravitational acceleration and the adiabatic expansion at half-time step in order to ensure that the formal accuracy of the code will be of second order. In the hydrodynamic step, the hydrodynamics is added to the baryonic matter by updating the density, velocity, and pressure with the changes calculated from the TVD-ES code using the density and the updated velocity and pressure. In the gravitational step, the gravitational acceleration is added to the hydrodynamically updated momentum only. In the expansion step, the adiabatic expansion is added to the hydrodynamically gravitationally updated momentum and the adiabatic cooling to the hydrodynamically updated pressure. The final updated total energy is, then, calculated from these updated momentum and pressure. The reason that the total energy itself does not go through the gravitational step is following: Suppose the total energy is updated in the gravitational step according to equation (3.3) and the thermal energy calculated by subtracting the kinetic energy from the total energy. Then, the error in including the gravitational effects could contaminate the thermal energy directly, if the gravitational energy is much larger than the thermal energy, say, if $W \gtrsim 10^{4} E_{\mathrm{th}}$ (where $W$ is the gravitational energy).

The gravitational force on the baryonic grid is calculated by finite differencing the potential fields at two adjacent grids. The gravitational force on each dark matter particle is calculated by linear interpolation of the forces at the eight neighboring grids using the cloud-in-cell scheme. The staggered mesh method (Melott 1986; Centrella et al. 1988) is adopted to achieve better spatial resolution. The advantages of using this scheme over a two-point finite difference approximation have been discussed extensively by Park (1991).

In order to handle discontinuities and structures accurately, all the numerical codes put the numerical diffusion in the mass flux as well as the momentum and energy fluxes. Here, let $D_{\rho k}$ be the numerical diffusive mass flux in the $k$ direction in our cosmological hydrodynamic code. We have modified the gravitational force term in the $x$-momentum equation by a factor of $\left[1-D_{\rho x} /\left(\rho u_{x}\right)\right]$, that in the $y$-momentum equation by $\left[1-D_{\rho y} /\left(\rho u_{y}\right)\right]$, and that in the $z$-momentum equations by $[1$ $\left.-D_{\rho z} /\left(\rho u_{z}\right)\right]$, in order to take account of the effective acceleration of the gravity acting on cells with the diffusive mass flux added. Note that this diffusive mass correction is somewhat different from that given in Cen (1992). The correction is small
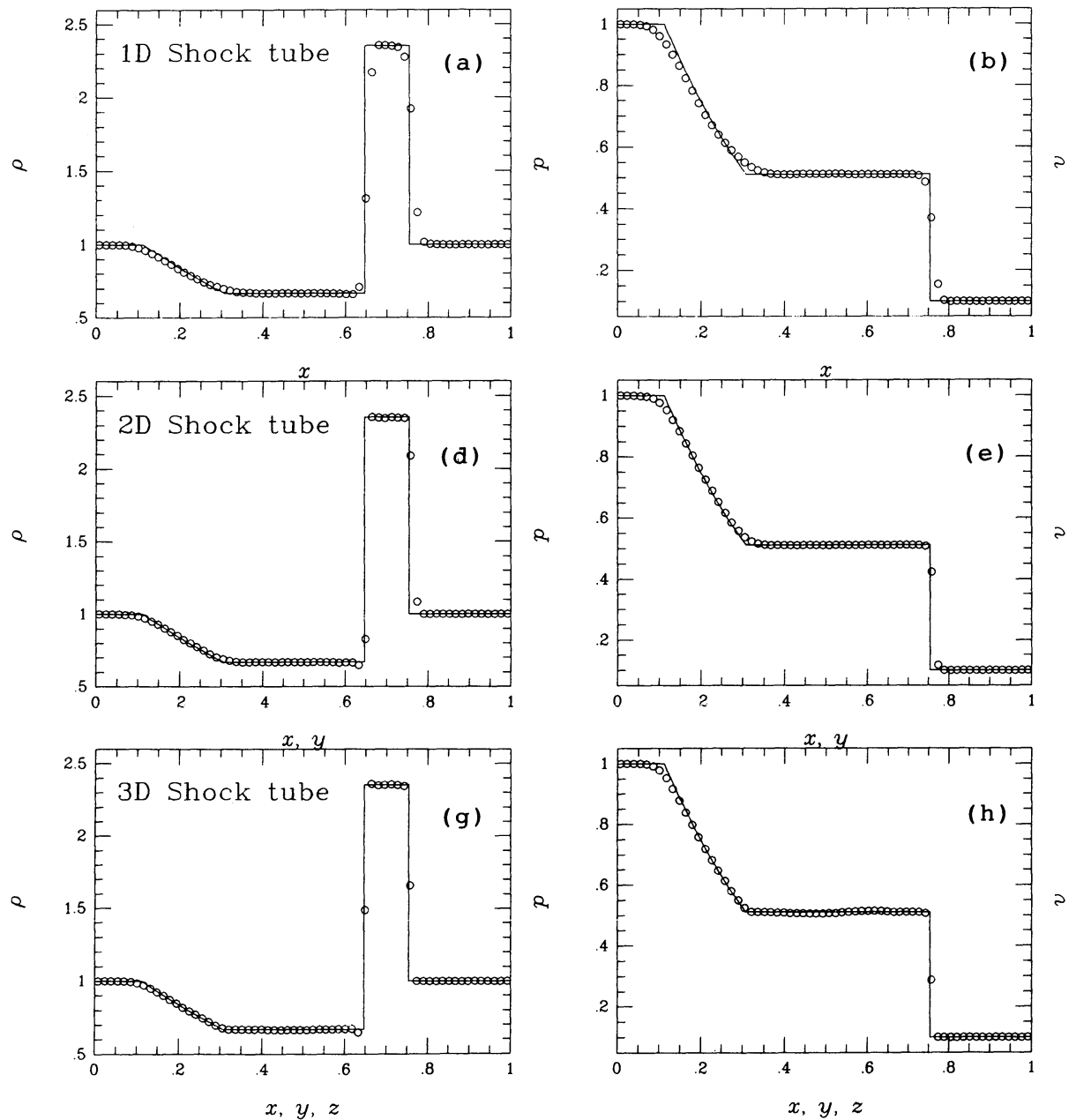
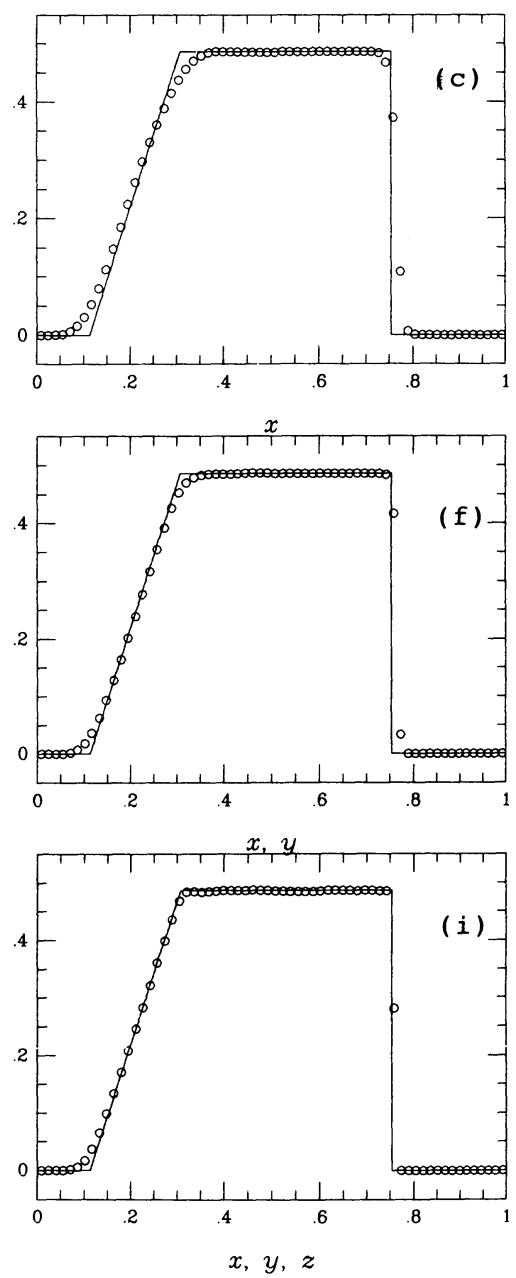

FIG. 1.-Standard shock tube test with TVD code. One-dimensional shock propagates along the line in the $x$-axis, two-dimensional shock propagates diagonally along the line $(0,0)$ to $(1,1)$ in the $x-y$ plane, and three-dimensional shock propagates diagonally along the line $(0,0,0)$ to $(1,1,1)$ in the $x y z$ cube. The numerical results (open circles) are plotted against the exact analytic solution (solid line) at $t=0.3$. 
in the smooth flow, $D_{\rho i} /\left(\rho u_{i}\right) \ll 1$, while it is close to unity around the shocks. In order to understand the effects of this diffusive correction in the gravitational force, let us look at the corresponding correction in the kinetic energy. The change in the kinetic energy of a cell during $\Delta t$ due to the gravity is $\rho u_{k} g_{k} \Delta t\left(g_{k}=-\partial \phi / \partial x_{k}\right)$. Around the shock, however, the mass flux $\rho u_{k}$ is modified by the numerical mass diffusive flux $D_{\rho k}$ to capture the shock correctly without generating nonphysical oscillations. Thus, the corrected change in the kinetic energy is $\left(\rho u_{k}-D_{\rho k}\right) g_{k} \Delta t$. This correction is required in any hydrodynamic code with self-gravity in order to conserve the total (kinetic, thermal, and gravitational) energy. Without this term, the change in the kinetic energy is either underestimated or overestimated for the shocks propagating under the gravitational field (see $\S 4$ for tests).

We should note that, with the correction added in the gravitational force term, the total momentum is not conserved. Instead, we could add an appropriate correction to the energy equation so that the correction due to the mass diffusion directly affects the thermal energy. However, we have chosen to make a small fractional error in the momentum while avoiding a large fractional error in the thermal energy which is more vulnerable to numerical contamination.
The time step in our cosmological code is determined by the two criteria: (1) the usual Courant condition, $\Delta t_{\text {courant }} \leq$ $C_{\text {Courant }} a \Delta x /\left(\left|u_{i}\right|+c_{s}\right)$ with $C_{\text {Courant }}=0.9$, and (2) $\Delta t_{\text {expansion }}$ satisfying $\Delta a=0.15$. The time step is $\Delta t=\min (\Delta t$ Courant $\left.\Delta t_{\text {expansion }}\right)$. Our tests indicate that, in the calculations with $128^{3}$ cells or smaller, $\Delta t_{\text {expansion }}$ is smaller than $\Delta t_{\text {Courant }}$ for most of the steps and determines the time step.

Our cosmological hydrodynamic code runs at the speed of $\sim 3.5 \times 10^{3}$ zones per second with one CPU on a Convex 220 or at $\sim 2.1 \times 10^{4}$ zones per second with one CPU on a Cray 2 .

\subsection{Cosmic Energy Equation}

The energy conservation for an ideal gas in expanding cosmological coordinates was derived in Peebles (1981). For gas with the adiabatic index of $\gamma=5 / 3$, the equation for the energy conservation can be written as

$$
\frac{d}{d t}(\mathscr{E}+W)+\frac{\dot{a}}{a}(2 \mathscr{E}+W)=0,
$$

where $\mathscr{E}$ and $W$ are the total (kinetic plus thermal) peculiar energy and the gravitational energy integrated over the computational periodic space (or the entire universe), respectively.
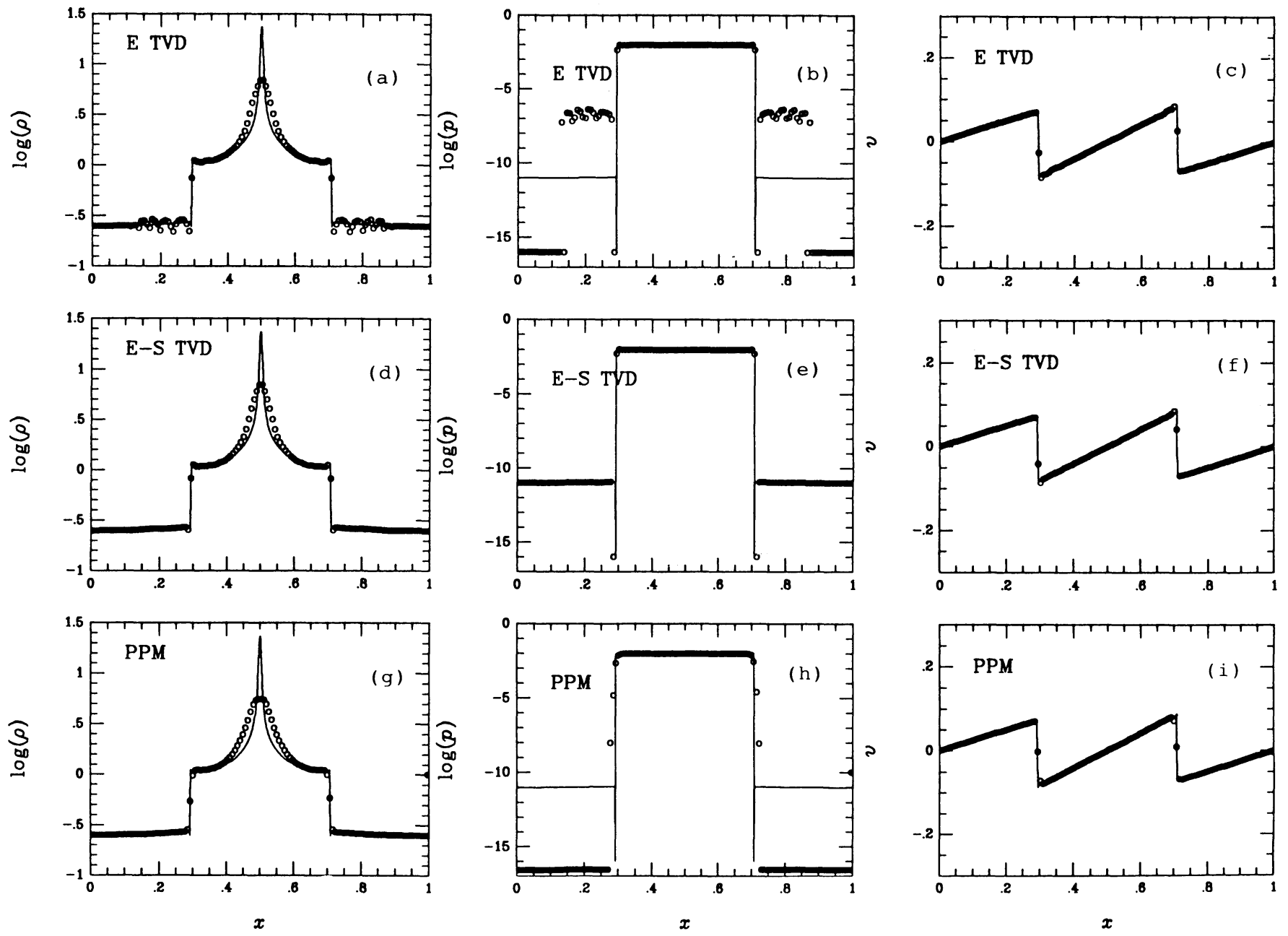

FIG. 2.-One dimensional caustic test with TVD-E, TVD-ES, and PPM codes. Two shocks are developed from the initial sinusoidal velocity field with peak value $1 / 2 \pi$, and the initial density and pressure are uniform with $\rho=1$ and $p=10^{-10}$. The open circles are results of each code with 128 cells, while the solid lines represent the results of TVD-ES code with 1024 cells, at $t=3$. 
One can show that this equation is also valid for a real viscous fluid, because the additional viscosity term in the total energy equation is conservative. In a real viscous fluid, shocks are not discontinuous jumps and the flow is continuous inside shocks.

We can follow Peebles (1980) to derive the equation for the energy conservation in the numerical simulations where the discontinuous jumps are captured with numerical dissipation. One can easily show that the cosmic energy equation (3.8) is also valid for the numerical simulations, only if the mass diffusive correction discussed in the previous subsection is added in the gravitational force.

The equation for the energy conservation (3.8) can be rewritten as

$$
a \mathscr{E}(a)-a_{0} \mathscr{E}\left(a_{0}\right)+\int_{a_{0}}^{a} \mathscr{E} d a=-\left[a W(a)-a_{0} W\left(a_{0}\right)\right],
$$

where $a$ and $a_{0}$ are the values of the expansion parameters at two different epochs. This simply shows that the increase of the total energy comes from the increase of the gravitational energy minus the adiabatic cooling due to the expansion. In order to check the accuracy of the energy conservation in our code, we define the conservation parameter $R$,

$$
R \equiv \frac{\left[a \mathscr{E}(a)-a_{0} \mathscr{E}\left(a_{0}\right)+\int_{a_{0}}^{a} \mathscr{E} d a\right]}{-\left[a W(a)-a_{0} W\left(a_{0}\right)\right]},
$$

which should remain unity if the energy conservation is perfect and we use it in the tests presented in the next section.

\section{NUMERICAL EXPERIMENTS}

\subsection{Shock Tube Test}

Figure 1 shows the results of a standard shock tube test calculated with the one, two- and three-dimensional TVD-E code. Initially, the flow is uniform $(\rho=1)$ and at rest $(v=0)$, while there is a pressure jump in the middle from $p=1$ to $p=0.1$. The calculations have been done with 64 cells, and the results shown are at $t=0.5$. In the one-dimensional calculation, the shock propagates along the line in the $x$-axis; in the two-dimensional calculation, along the line $(0,0)$ to $(1,1)$ in the $x-y$ plane; and in the three-dimensional calculation, along the
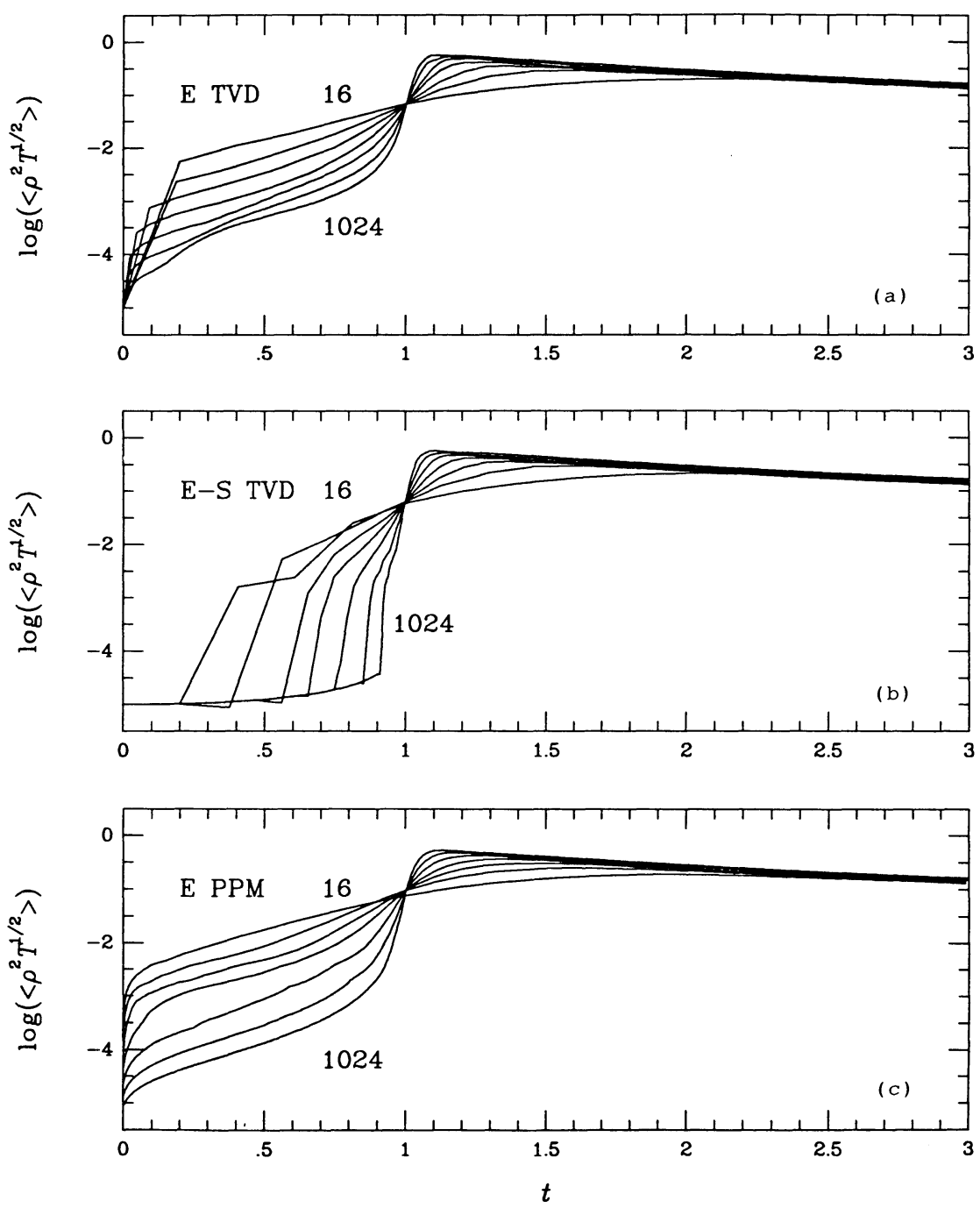

FIG. 3.-Evolution of the total luminosity from the one-dimensional caustic tests shown in Fig. 2 as a function of time. Each line corresponds to the luminosity with different resolutions $\left(n_{\text {cell }}=16,32,64,128,256,512,1024\right)$. 
line $(0,0,0)$ to $(1,1,1)$ in the $x y z$ cube. The shock is typically resolved within two to three cells as well as the contact discontinuity. One thing noticeable is that there is an improvement (instead of a decay) of quality in the multidimensional calculations.

The above test has been done with the steepening technique for the contact discontinuity suggested by Harten (1983). The steepening scheme was designed to work only on the field involving the entropy mode and have effects only on the contact discontinuity. Without the steepening, a contact discontinuity is typically spread over six to seven cells. While the steepening makes the contact discontinuity look good, we turned off the steepening in our three-dimensional code due to the following reasons: the contact discontinuities form only when there are initial discontinuities. In the three-dimensional cosmological simulations for which we plan to apply our code, we expect that the initial condition does not involve any discontinuity, so no contact discontinuity will form.

\subsection{One-dimensional Hydrodynamic Caustics}

The formation of one-dimensional caustics has been calculated from the initial sinusoidal velocity field with the peak value $1 / 2 \pi$. The initial density and pressure has been set to be uniform with $\rho=1$ and $p=10^{-10}$. Hence, the initial peak velocity corresponds to a Mach number of $1.2 \times 10^{4}$. The calculations have been done with 128 cells using the TVD-E, TVD-ES, and PPM codes and the results at $t=3$ are shown in Figure 2. They are compared with that of the TVD-ES with 1024 cells. Note that only the TVD-ES code can correctly follow the adiabatic changes in the preshock region. On the other hand, the results of the TVD-E code shows oscillations in density and substantial preshock heating. The PPM code reproduces the correct density profile without oscillations, but the thermal energy becomes negative and must be reset to be an arbitrary small number in the preshock region.

We also calculate the time evolution of the total luminosity $\left(\int \rho^{2} T^{1 / 2} d V\right)$ generated by the one-dimensional caustics shown in Figure 2 by assuming simple bremsstrahlung emissivity. In Figure 3, the total luminosity is plotted against the time for the calculation done with different resolutions using the TVD-E, TVD-ES, and PPM codes. The results show that even with the highest resolution the TVD-E and PPM codes incorrectly generate a substantial amount of luminosity well before the shock is formed around $t=1$, proving the superiority of the TVD-ES code for problems involving extremely supersonic flows.
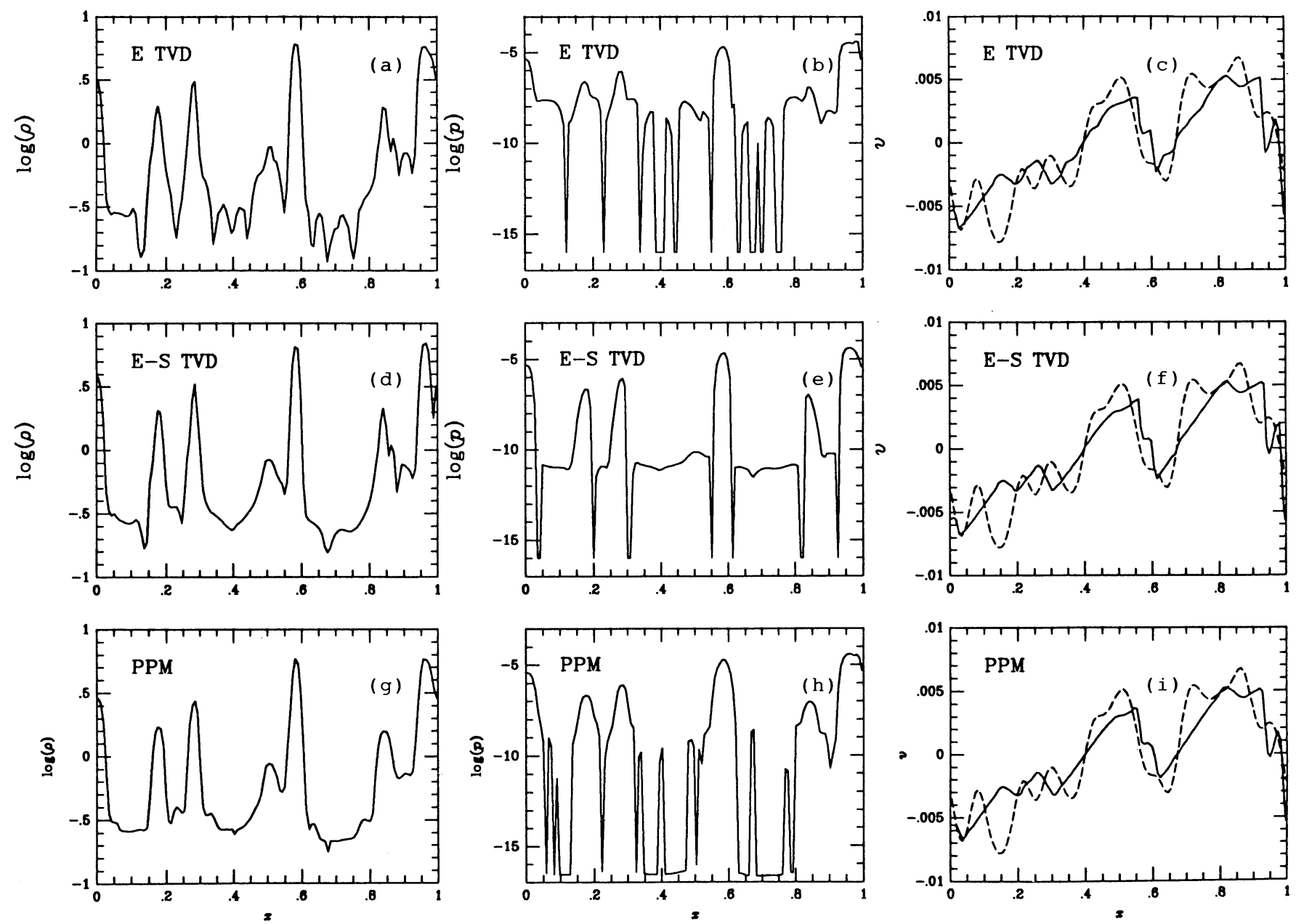

Fig. 4.-One-dimensional random velocity field test with TVD-E, TVD-ES, and PPM codes. The initial random velocity is shown by the dashed line, and the initial density and pressure are uniform with $\rho=1$ and $p=10^{-10}$. The line shows the results at $t=20$. 


\subsection{One-dimensional Hydrodynamic Flow with Random Velocity Field}

The evolution of a one-dimensional flow with a random initial velocity has been followed. The initial velocity is given with the dashed line in Figure 4, and the initial density and pressure have been set to be uniform with $\rho=1$ and $p=10^{-10}$. The maximum Mach number of the velocity is about 620 . The calculations have been done with 128 cells using the TVD-E, TVD-ES, and PPM codes and the results at $t=20$ are shown in Figure 4. The results show that the TVD-ES code can resolve the shocks without any noticeable preshock heating and the density in the expanding region seems to behave well. The pressure in one cell immediately next to the shock tends to go negative. But this does not have any significance in terms of total entropy generation. However, as in the one-dimensional hydrodynamic caustic test, the TVD-E code generates a substantial amount of heating in the expanding, unshocked region, while the PPM code tends to make the expanding region have a negative thermal energy.
4.4. One-dimensional Cosmological Pancake Collapse

In order to test the cosmological hydrodynamic code with the TVD-ES scheme including gravity in comoving coordinates, we have calculated the collapse of a one-dimensional pancake (i.e., the Zel'dovich pancake problem) in a purely baryonic universe with $\Omega=1$ and $h=\frac{1}{2}$. Initially at $a_{i}=1$ (which corresponds to $z_{i}=20$ in this test), a sinusoidal velocity field with the peak value $0.65 /\left(1+z_{i}\right)$ in the normalized units has been imposed in a box with the comoving size $64 h^{-1} \mathrm{Mpc}$, so that the shocks form at $a=10.5$ (or $z=1$ ). The baryonic density and pressure have been set to be uniform with $(\rho$, $p)=\left(1,6.2 \times 10^{-8}\right)$ in the normalized units which correspond to the temperature $10^{4} \mathrm{~K}$ in the physical units. The calculation has been done with 128 cells and with/without the mass diffusive correction term for the gravitational force discussed in $\S 3.1$. In Figure 5, the simulated pancakes around central 20 cells at present $(a=21$ or $z=0)$ are plotted for $n_{\text {cell }}=128$ and are compared with that calculated with 1024 cells and with mass diffusive correction (i.e., our best simulation). The shocks
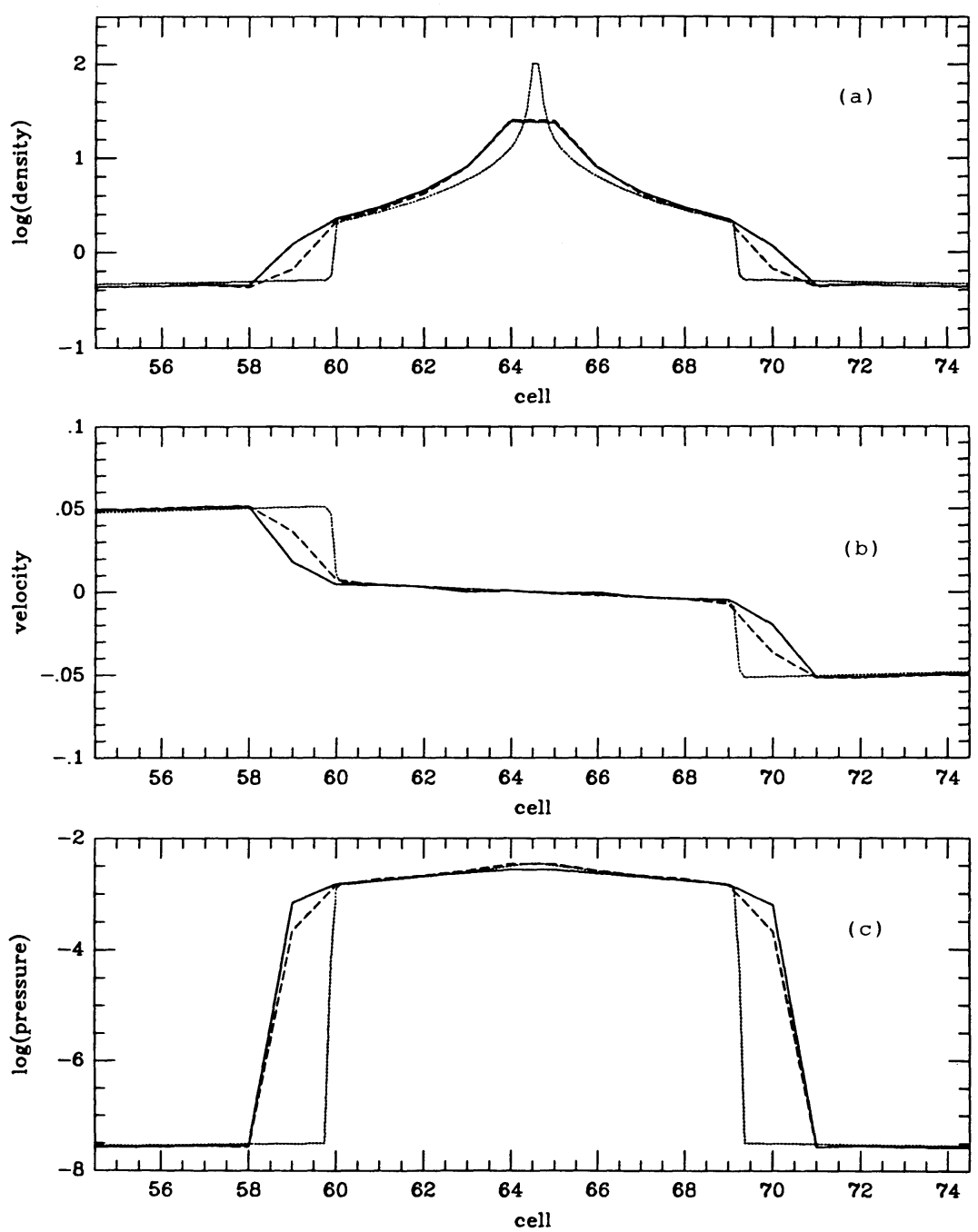

Fig. 5.-One-dimensional gravitational collapse of a pancake in the comoving coordinates with TVD-ES code. Initially at $a=1$, a sinusoidal velocity field with a peak value $3.1 \times 10^{-2}$ is imposed and the density and pressure are uniform with $\rho=1$ and $6.2 \times 10^{-8}$. The solid and the dashed lines show the flow structure at $a=21$ with and without the diffusive correction, respectively, and with $n_{\text {cell }}=128$. The dotted lines represent the result with the diffusive correction and with $n_{\text {cell }}=1024$. 
are resolved within two cells, and there is no numerical heating in the preshock region. The shocks at the lower resolution are farther out than those at the higher resolution, but the difference is only about one cell width. As expected, the diffusive correction affects mostly the shocking zones, but the difference is negligible indicating the fairness of our scheme for the mass diffusive correction.

In order to test the energy conservation with our cosmological hydrodynamic code, we have calculated the conservation parameter $(R)$, the total gravitational energy $(W)$, the total kinetic energy $\left(\mathscr{E}_{\text {kin }}\right)$, and the total thermal energy $\left(\mathscr{E}_{\text {th }}\right)$ in the above one-dimensional pancake collapse test done with different resolutions. The resulting values at present $(a=21$ or $z=0$ ) are plotted against the resolution in Figure 6 . The inclusion of the mass diffusive correction term improves not only the conservation of total energy but also the convergence of the code. From Figure $6 a$ we see that, with our TVD-ES code with the diffusive mass correction, the error in energy conservation is $\sim 1 \%$ at $n_{\text {cell }}=32$ and declines to $\sim 0.01 \%$ at $n_{\text {cell }}=1024$. Note that the one-dimensional pancake collapse test requires at least 32 cells (or $\Delta x<\lambda / 32$ ) in order to achieve the proper convergence with the initial conditions described in the above paragraph.
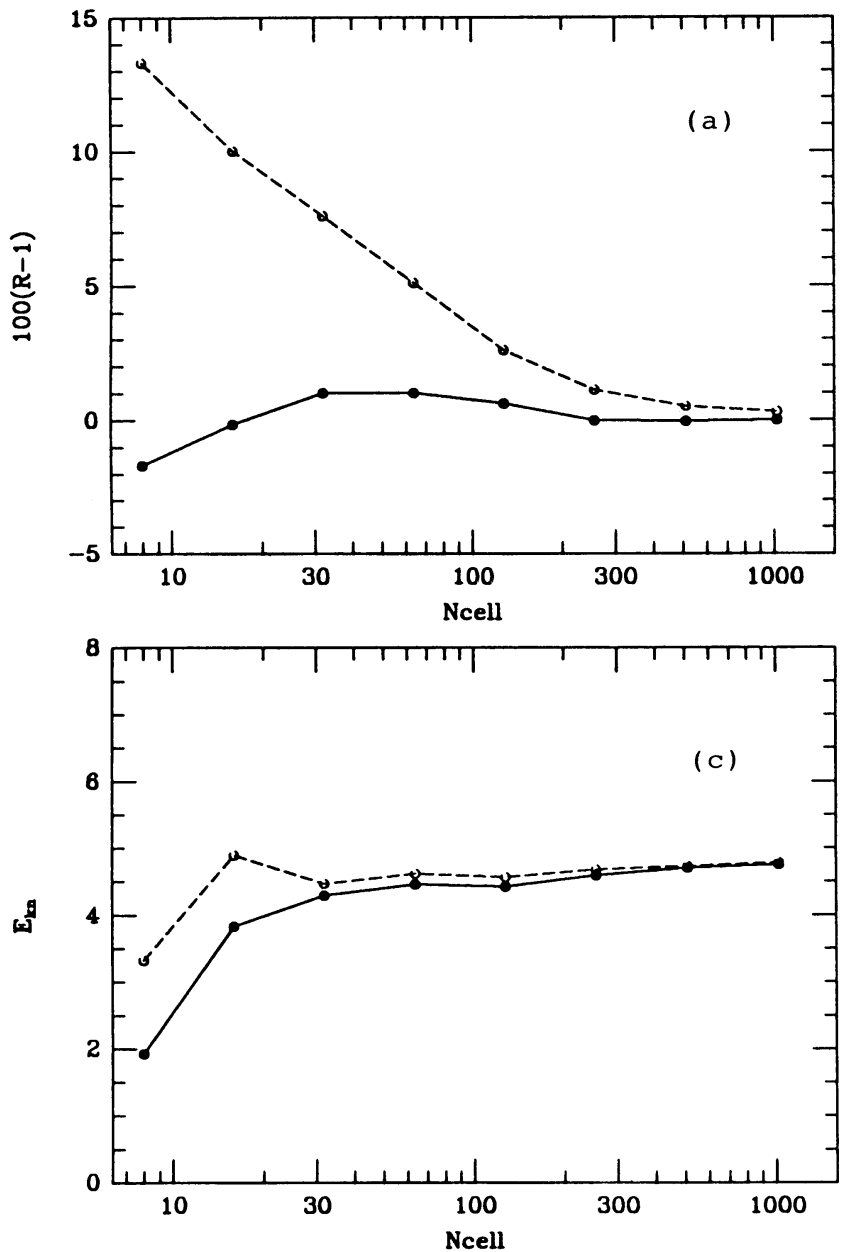

\subsection{Three-dimensional Adiabatic CDM Universe}

We have calculated the adiabatic evolution of a purely baryonic universe but with an initial CDM power spectrum. The purpose of such a test calculation is to focus on the hydrodynamic properties of our code, even though the code can handle the dark matter as well as the baryonic matter. Also, the dark matter part of our code behaves similarly to the cosmological $N$-body code which was already studied extensively (see, e.g., Efstathiou et al. 1985). Random Gaussian initial conditions have been generated from the initial power spectrum using the process described in Weinberg \& Gunn (1990) with the normalization that the mass fluctuation in a sphere of radius $8 h^{-1}$ $\mathrm{Mpc}$ is $\sigma_{8}$. The values of the usual cosmological parameters are $\Omega=\Omega_{b}=1 ., h=0.5$, and $\sigma_{8}=1$, and the size of the computational cube is $L=64 h^{-1} \mathrm{Mpc}$. The universe has been evolved from $a=1$ (or $z=20$ ) to $a=21$ (or $z=0$ ). Four sets of calculations have been done using the TVD-E and TVD-ES codes with/without the mass diffusive correction, and $128^{3}$ cells have been used.

Figure 7 shows the volume-weighted histogram of the baryonic temperature at $z=0$ for the four simulations. As expected, the volume occupied by the high-temperature
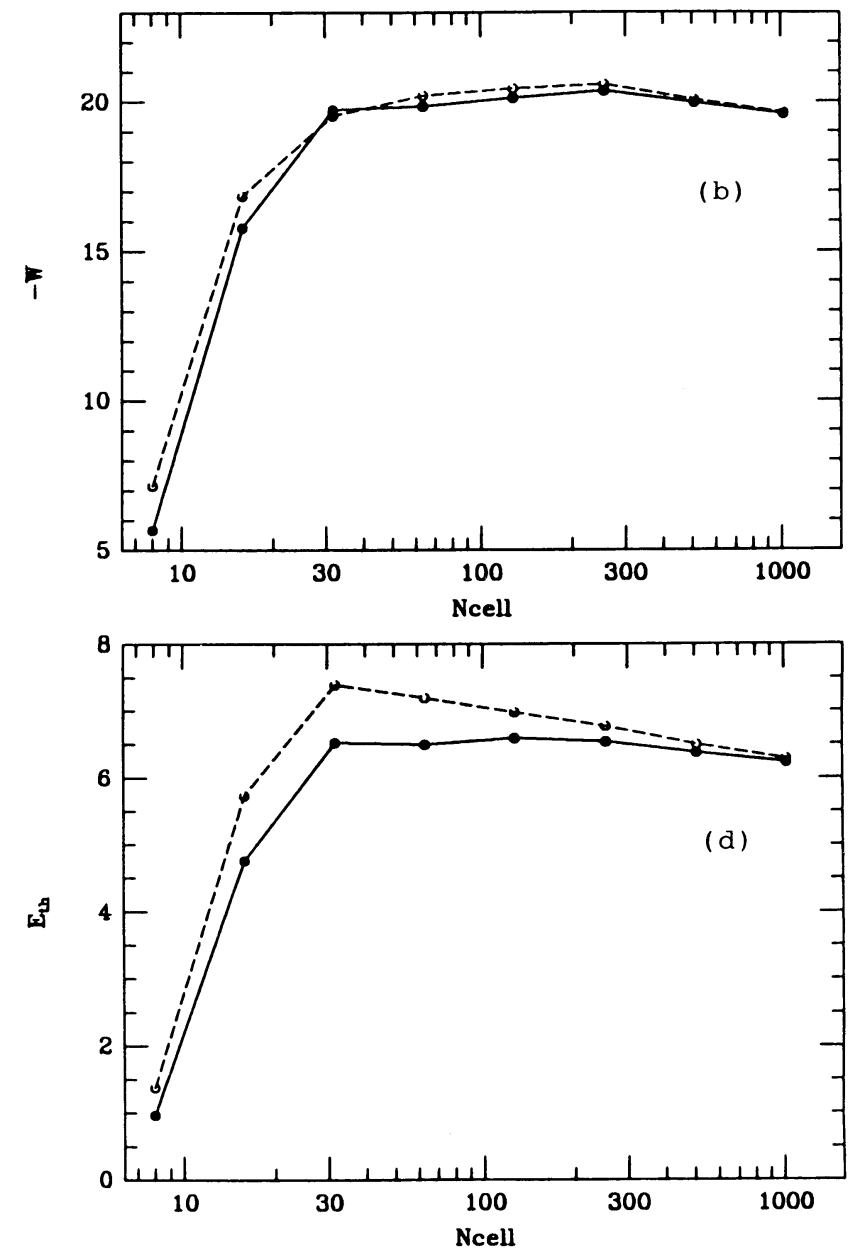

Fig. 6. - Conservation parameter, $R$ (see the text for the definition), gravitational energy, $W$, kinetic energy, $\mathscr{E}_{\text {kin }}$, and thermal energy, $\mathscr{E}_{\text {th }}$ of the one-dimensional pancake simulations shown in Fig. 5 for different resolutions $\left(n_{\text {cell }}=8,16,32,64,128,256,512,1024\right)$ at $a=21$. The solid and the dashed lines show the values with and without the diffusive correction, respectively. 
TVDES, TVDES,

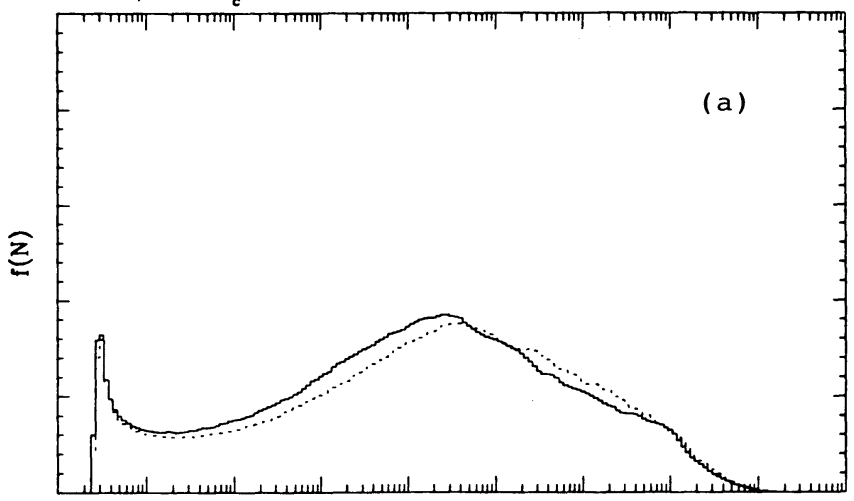

TVDE, TVDE

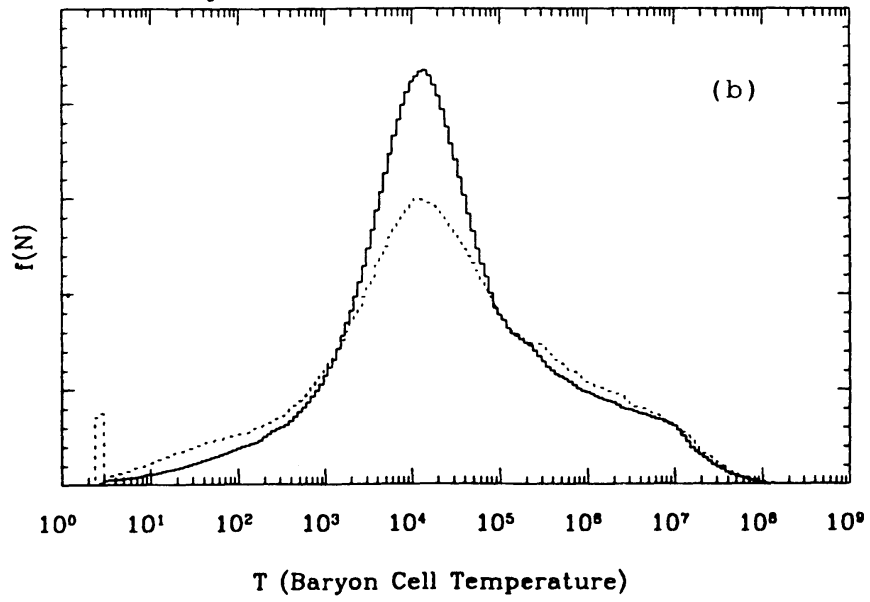

FIG. 7.-Volume-weighted temperature histogram at present $(a=21)$ for the three-dimensional simulations of a purely baryonic, adiabatic universe with the CDM initial power spectrum. The top panel is for the simulations with TVD-ES code and the bottom panel with TVD-E code. The solid lines are for the simulations with the diffusive correction and the dotted lines without the diffusive correction.

regions with $T \gtrsim 10^{6} \mathrm{~K}$ is comparable in both codes. However, the histogram shows that the TVD-E code generates spurious numerical heating in the low-temperature regions with $T \gtrsim$ $10^{4} \mathrm{~K}$ and heats up those regions up to around $T \sim 10^{4} \mathrm{~K}$. But in the TVD-ES code, the spurious heating stays minimal and the fraction of the volume in cold, unshocked regions is larger than that in the TVD-E code. The radical difference in the fractional volume of gas at $T \sim 10^{4} \mathrm{~K}$ shows the importance of minimal leaks of energy from kinetic to thermal form. The diffusive correction term tends to lower the temperature in both the TVD-ES and TVD-E codes, but its effect is not very significant especially in the TVD-ES code.

The difference of the TVD-ES and TVD-E codes is also obvious in the contour plot of the volume with given temperature and density at $z=0$. As can be seen in Figures $8 a$ and $8 b$, the volume of the high-density $(\rho / \bar{\rho} \gtrsim 3)$-high-temperature $\left(T \gtrsim 10^{6} \mathrm{~K}\right)$ regions which are occupied by strongly shocked gas is comparable in both codes. Also, both codes produce a similar amount of the volumes of the medium-density $(1 \lesssim$ $\rho / \bar{\rho} \lesssim 3)$-medium-temperature $\left(10^{4} \lesssim T \lesssim 10^{6} \mathrm{~K}\right)$ regions and the medium- to low-density $(\rho / \bar{\rho} \lesssim 3)$-high-temperature $(T \gtrsim$ $\left.10^{6} \mathrm{~K}\right)$ regions. The difference mostly occurs in the lowdensity-low-temperature regions: the TVD-E code heats a sig- nificant portion of the regions with $\rho / \bar{\rho} \lesssim 1$ and $T \lesssim 10^{4} \mathrm{~K}$ to $T \sim 10^{4} \mathrm{~K}$. For comparison, we plot in Figure $8 c$ the same contour diagram from the same calculation, but using the code described in Cen (1992). The volume of the high-densityhigh-temperature regions is similar to that in Figures $8 a$ and $8 b$, but the numerical heating of the gas with low temperature $T \lesssim 10^{4} \mathrm{~K}$ is very severe in this code and tends to heat up all the low-temperature gas to $T \sim 10^{4} \mathrm{~K}$.

The density, velocity, and temperature structures along an arbitrary chosen line of sight parallel to the $x$-axis of the computational box for the four simulations are shown in Figure 9. Here, the density and velocity are given in the normalized units and the temperature in the physical units. The results show that the velocity is very similar in the simulations with both codes as well as the density and temperature of high-densityhigh-temperature regions. Once again, the heating of the unshocked gas in the low-density region with $T \lesssim 10^{4} \mathrm{~K}$ is evident in the simulations with the TVD-E code. The mass diffusive correction tends to smooth somewhat the velocity around the shock and reduce the temperature of the shocked gas in both the TVD-ES and TVD-E codes.

Figures $10 a$ and $10 b$ show the temperature contour plots at $z=0$ of a slice with one cell thickness $\left(0.5 h^{-1} \mathrm{Mpc}\right)$ in the simulations using the TVD-ES and TVD-E codes with the mass diffusive correction. The regions with the high temperature $\left(T \gtrsim 10^{6} \mathrm{~K}\right)$ almost coincide in both calculations. However, the plot for the TVD-ES code shows a large fraction of the volume in regions of cold gas $\left(T \lesssim 10^{4} \mathrm{~K}\right)$, while such regions disappear in the plot for the TVD-E code due to the artificial numerical heating. Also, one can see that, especially in the plot for the TVD-ES code, the postshock gas and the unshocked IGM are distinctively separated by the wellresolved shocks.

Finally, we plot in Figure 11 the time evolution of the conservation parameter, $R$, the total gravitational energy, $W$, the total kinetic energy, $\mathscr{E}_{\text {kin }}$, and the total thermal energy, $\mathscr{E}_{\text {th }}$ for the four simulations each with $n_{\text {cell }}=128$. The plots clearly demonstrate the improvement of the energy conservation with the mass diffusive correction in both the TVD-ES and TVD-E codes. In the code with the mass diffusive correction, energy is conserved within $\sim 4 \%$ even in the extremely nonlinear final state. But, the difference between the TVD-ES code and the TVD-E code changes $R$ and total energies very little, because it affects only the low-density-low-temperature regions while the energy mostly resides in the high-density-high-temperature regions.

\section{SUMMARY}

We have described a new cosmological hydrodynamic code which is based on the Harten (1983) total variation diminishing scheme. It is a flux-based Eulerian code with second-order accuracy in space and time. It captures shocks within two to three cells without generating oscillations by limiting the numerical flux according to the TVD scheme instead of adding a simple artificial viscosity. According to our tests, the numerical resolution and the convergence are nearly as good as the well-known PPM code, yet the computational speed is substantially faster.

While incorporating the TVD scheme into the cosmological code, we have made several important improvements over the previously developed hydrodynamic code with self-gravity for cosmological problems (Ryu et al. 1990; Cen 1992; Katz et al. 1992). First, with the TVD-ES scheme, we have eliminated the 


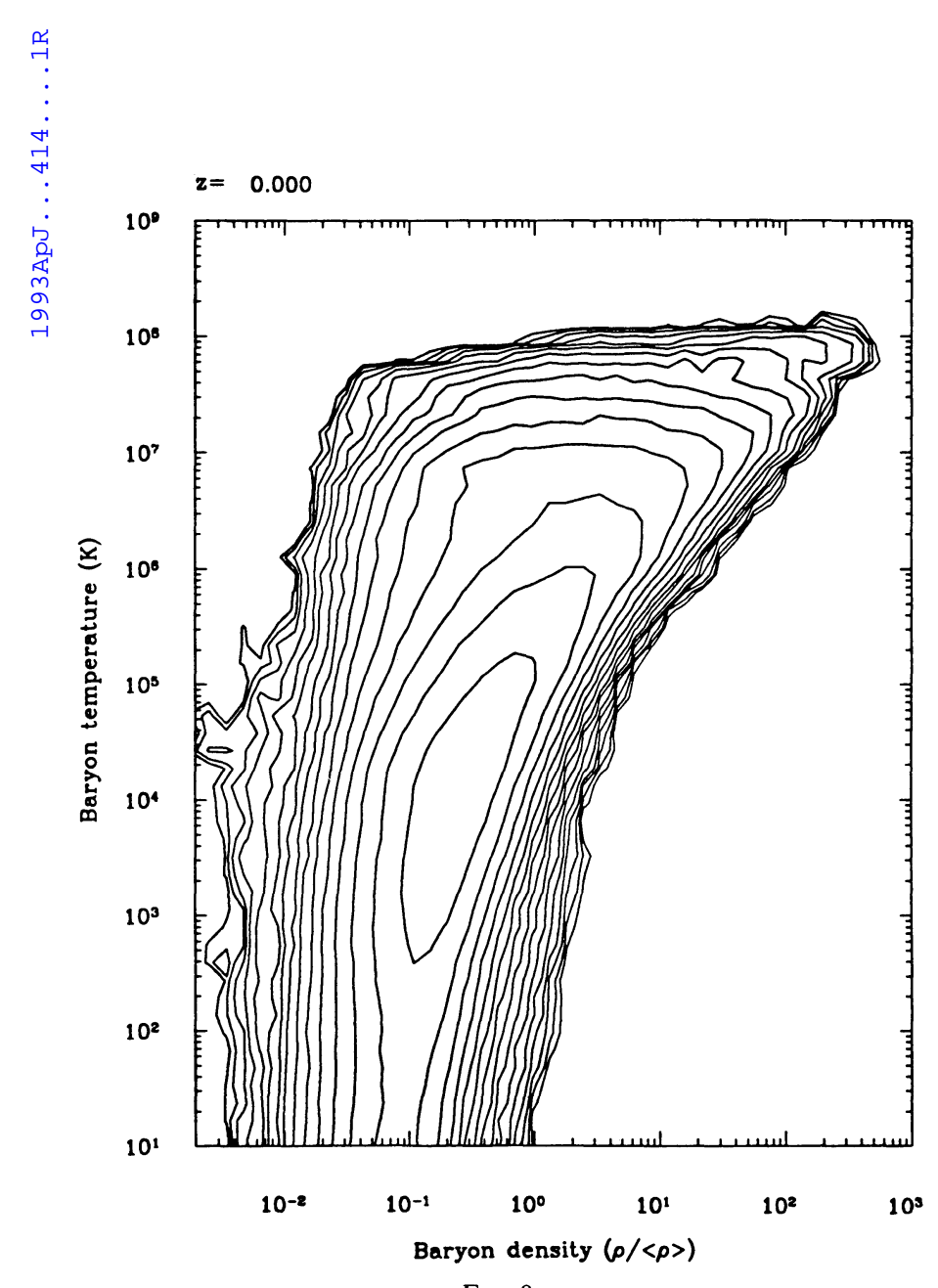

FIG. $8 a$

$\mathrm{z}=0.000$

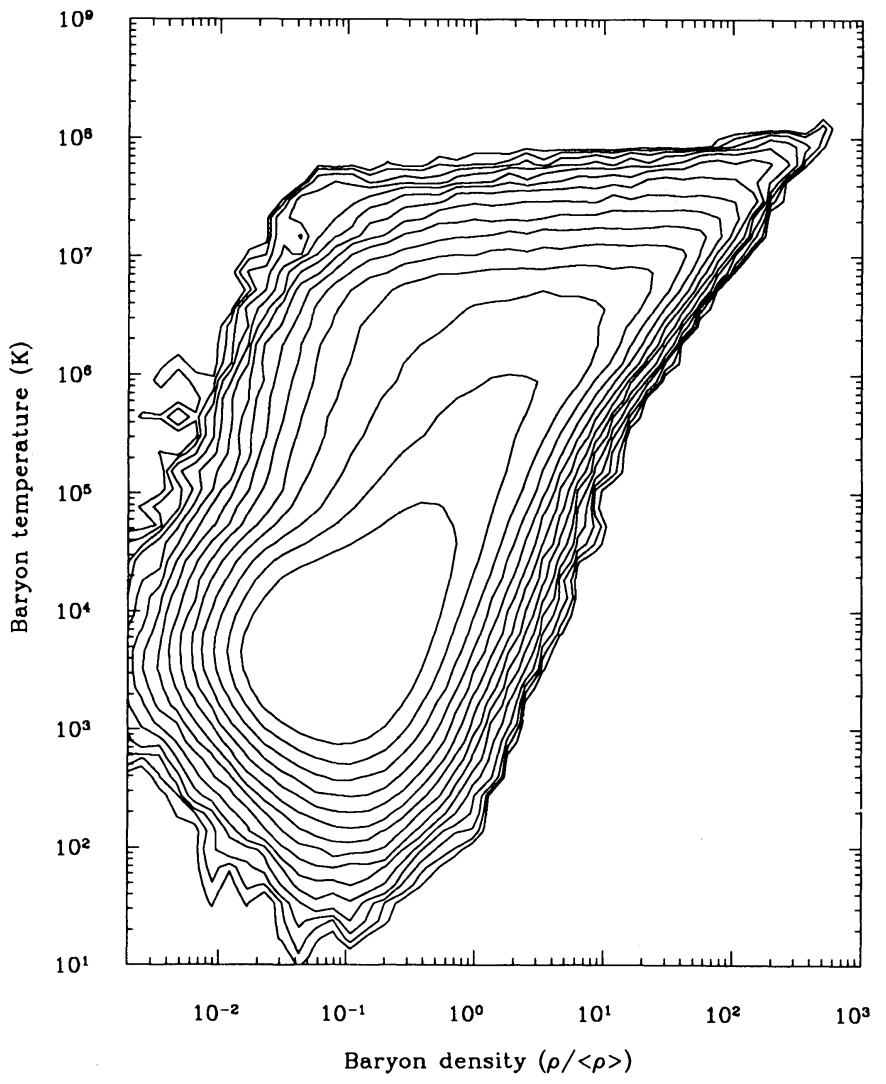

FIG. $8 c$

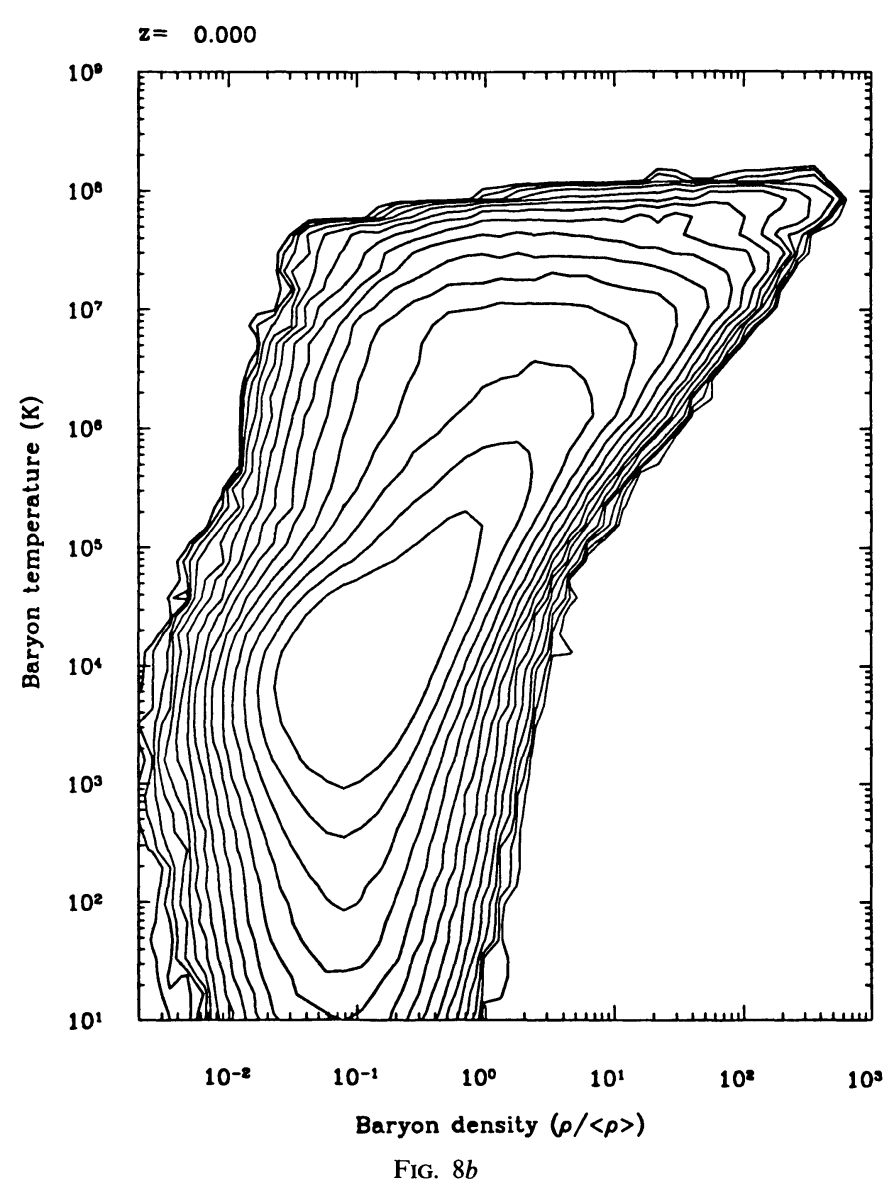

FIG. 8.-Contour plot of the volume with given temperature and density at present for the three-dimensional simulations of a purely baryonic, adiabatic universe with the CDM initial power spectrum. The plots are for the calculations $(a)$ with TVD-ES with diffusive correction and $(b)$ with TVD-E with diffusive correction. For comparison, the same contour from the same calculation with the code described in Cen (1992) is plotted in (c). 

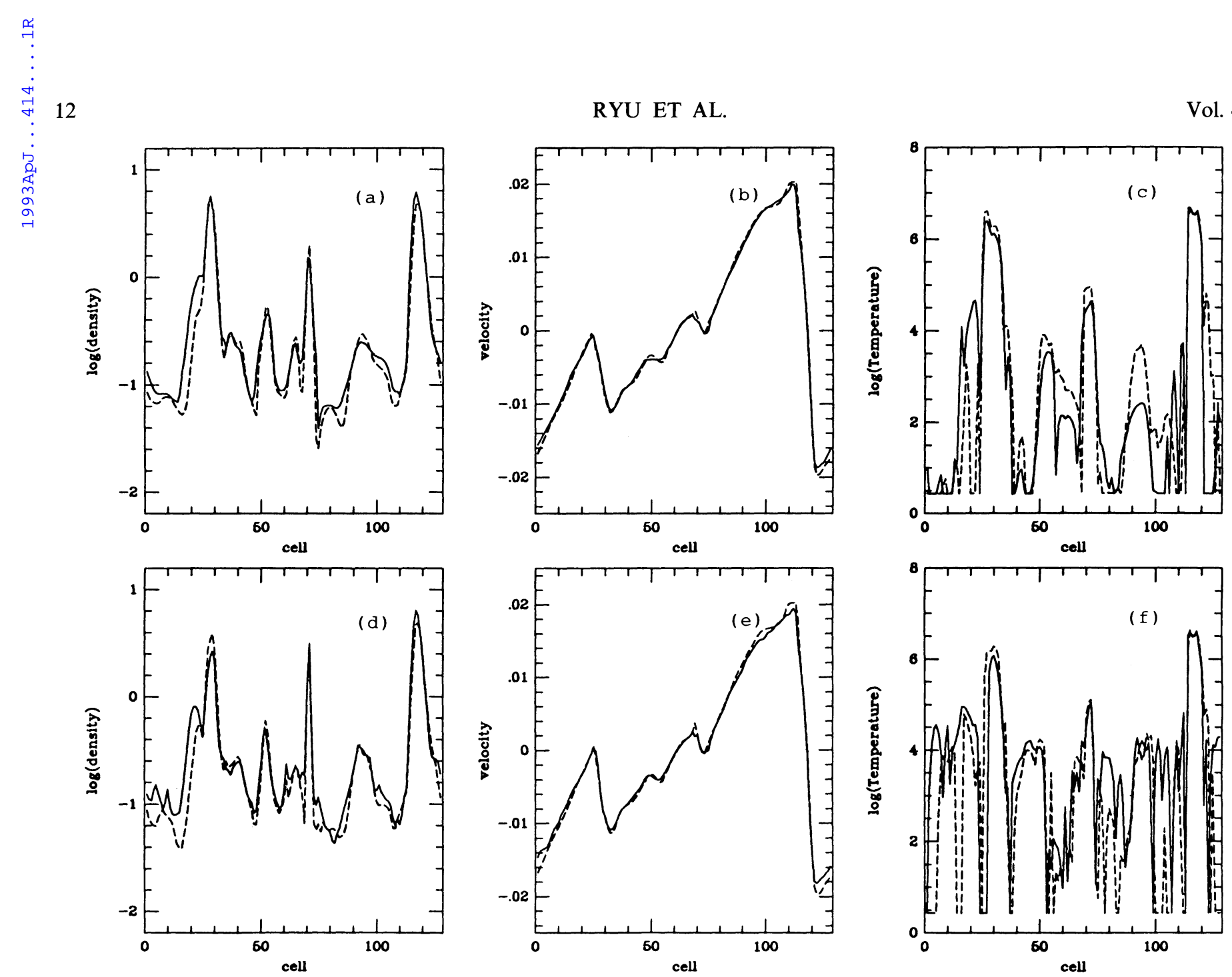

FIG. 9.-Flow structure along a line of sight at present for the three-dimensional simulations of a purely baryonic, adiabatic universe with the CDM initial power

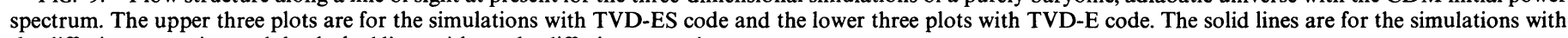
the diffusive correction and the dashed lines without the diffusive correction.

artificial numerical heating around the extremely supersonic flows where the bulk kinetic energy is much greater than the thermal energy. This has been achieved by following the adiabatic changes of the thermal energy using the modified entropy equation (2.4) instead of using the total energy equation (2.3) in the region free of shocks. Second, we have prevented the leakage of the gravitational energy into the thermal energy in regions of supersonic flow where the gravitational energy is comparable to the bulk kinetic energy and much larger than the thermal energy. This has been done by including the effects of the gravitational force only to the momentum and kinetic energy and keeping the thermal energy rather than solving the conservation of the total (thermal and kinetic) energy. Finally, a correction due to the mass diffusion under the gravitational field has been added in the gravitational force term in order to obtain better conservation of the total (thermal, kinetic, and gravitational) energy and to satisfy the cosmic energy equation (Peebles 1980). These improvements could be substantial for astrophysical gas flows which move supersonically under the self-gravity.

Numerical experiments presented in $\S 4$ have illustrated how much the code improves the results. In general, the TVD-ES code can correctly follow the adiabatic changes of extremely supersonic flows with a Mach number larger than $\sim 10^{4}$, while the TVD-E code generates artificial heating in the supersonically expanding, low-density region. The mass diffusive correction to the gravitational force around shocks improves the conservation of total (gravitational, kinetic, and thermal) energy dramatically. In the one-dimensional Zel'dovich pancake problem, an energy accuracy of $\sim 1 \%$ is achieved for $n_{\text {cell }}=32$ per unit wavelength and the accuracy reaches $\sim 0.01 \%$ for $n_{\text {cell }}=1024$. For a three-dimensional cosmological simulation, the error in the energy conservation is within $\sim 4 \%$. Without this correction, the total kinetic energy and the total thermal energy in the entire computational box are bigger than the total gravitational energy. This indicates that the increase in the kinetic energy of a flow element undergoing a shock while infalling under the gravitational potential will be overestimated without the mass diffusive correction.

We expect the difference between the TVD-ES code and the TVD-E code would be somewhat less significant in more realistic simulations where the radiation is allowed to be generated by the hot gas and its transfer is included, because the photoionization could heat the gas, say, up to $\sim 10^{4} \mathrm{~K}$. Inclusion of these physical processes in our cosmological code is underway and will be presented in separate papers. 
64.

in'

in:

In!

InI

48.

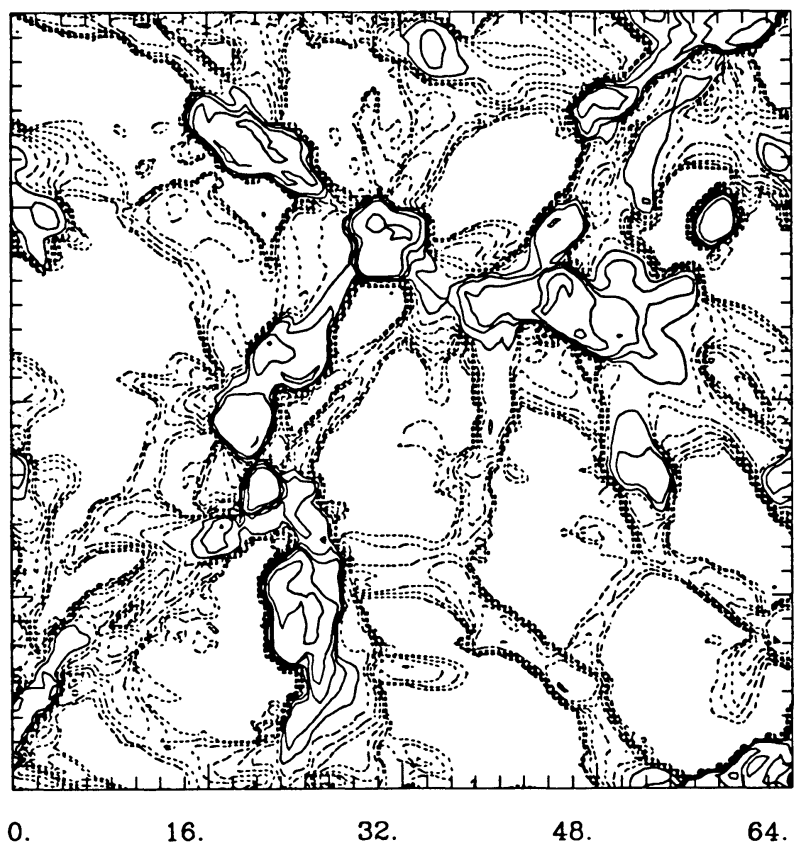

Bayonic Temperature Contour

solid contour: $\left(5^{1 / 2}\right) 10^{6} \mathrm{~K}, \mathrm{i}=0,1,2, .$.

dotted contour: $\left(5^{-1 / 2}\right) 10^{\circ} \mathrm{K}, \mathrm{i}=1,2, \ldots$

FIG. $10 a$
64.

48.

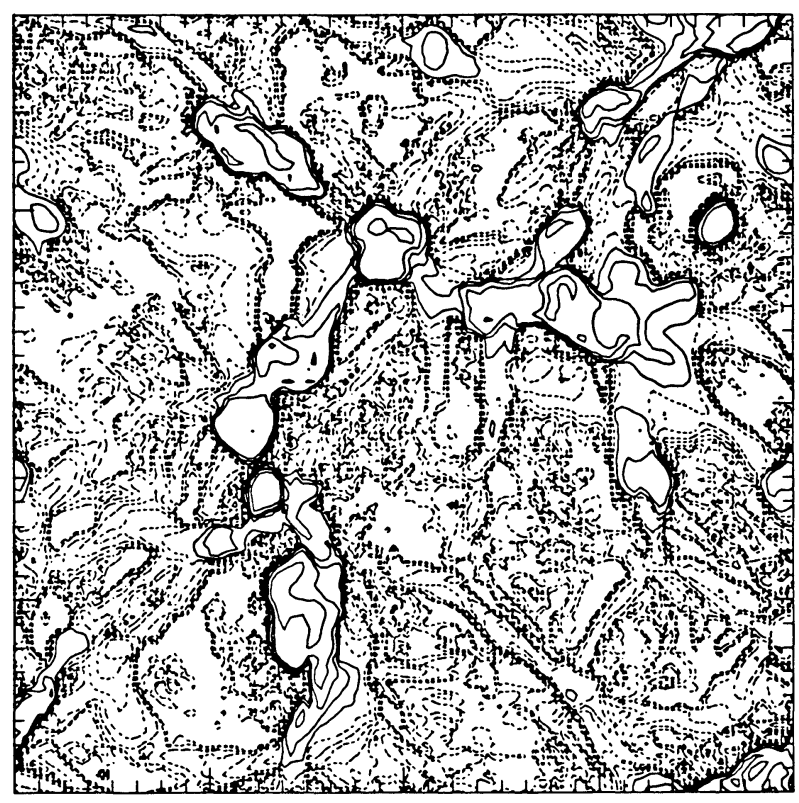

0 .

16.

32.

48.

64.

Bayonic Temperature Contour

solid contour: $\left(5^{1 / 2}\right) 10^{6} \mathrm{~K}, \mathrm{i}=0,1,2, \ldots$

dotted contour:(5-1/2) $10^{6} \mathrm{~K}, \mathrm{i}=1,2, \ldots$

Fig. $10 b$

FIG. 10.- Temperature contour plots of a slice with one cell width $\left(0.5 h^{-1} \mathrm{Mpc}\right)$ at present for the three-dimensional simulations of a purely baryonic, adiabatic universe with the CDM initial power spectrum. The plots are for the calculations $(a)$ with TVD-ES with diffusive correction and $(b)$ with TVD-E with diffusive correction.

We would like to thank Jeremy Goodman for helpful discussions. The work by D. R. was in part supported by David and Lucille Packard Foundation Fellowship through Jeremy
Goodman at Princeton University. The work by J. P. O., H. K., and R. Y. C. was supported in part by NASA through grant NAGW-2448 and NSF through grant AST 91-08103.

\section{APPENDIX A}

\section{EIGENVALUES AND EIGENVECTORS FOR THE HYDRODYNAMIC EQUATIONS}

The hydrodynamic equations in (2.1)-(2.3) are written in the vector form as

$$
\begin{gathered}
\partial_{t} \boldsymbol{q}+\partial_{x} \boldsymbol{F}_{x}+\partial_{y} \boldsymbol{F}_{y}+\partial_{z} \boldsymbol{F}_{z}=0, \\
\boldsymbol{q}=\left(\begin{array}{c}
\rho \\
\rho v_{x} \\
\rho v_{y} \\
\rho v_{z} \\
E
\end{array}\right), \quad \boldsymbol{F}_{x}=\left(\begin{array}{c}
\rho v_{x} \\
\rho v_{x}^{2}+p \\
\rho v_{x} v_{y} \\
\rho v_{x} v_{z} \\
(E+p) v_{x}
\end{array}\right), \quad \boldsymbol{F}_{y}=\left(\begin{array}{c}
\rho v_{y} \\
\rho v_{x} v_{y} \\
\rho v_{y}^{2}+p \\
\rho v_{y} v_{z} \\
(E+p) v_{y}
\end{array}\right), \quad \boldsymbol{F}_{z}=\left(\begin{array}{c}
\rho v_{z} \\
\rho v_{x} v_{z} \\
\rho v_{y} v_{z} \\
\rho v_{z}^{2}+p \\
(E+p) v_{z}
\end{array}\right),
\end{gathered}
$$

where the equation of state is given by $E=p /(\gamma-1)+\rho\left(v_{x}^{2}+v_{y}^{2}+v_{z}^{2}\right) / 2$.

With the vector, $\boldsymbol{q}$, and the flux functions, $\boldsymbol{F}_{x}(\boldsymbol{q}), \boldsymbol{F}_{y}(\boldsymbol{q})$, and $\boldsymbol{F}_{z}(\boldsymbol{q})$, the Jacobian matrices, $\boldsymbol{A}_{x}(\boldsymbol{q})=\partial \boldsymbol{F}_{x} / \partial \boldsymbol{q}, \boldsymbol{A} \boldsymbol{y}(\boldsymbol{q})=\partial \boldsymbol{F}_{y} / \partial \boldsymbol{q}$, and $\boldsymbol{A}_{z}(\boldsymbol{q})=\partial \boldsymbol{F}_{z} / \partial \boldsymbol{q}$, are formed. The system of equations in (A1) is called hyperbolic, if all the eigenvalues of the Jacobian matrix are real and the corresponding set of right eigenvectors is complete. 

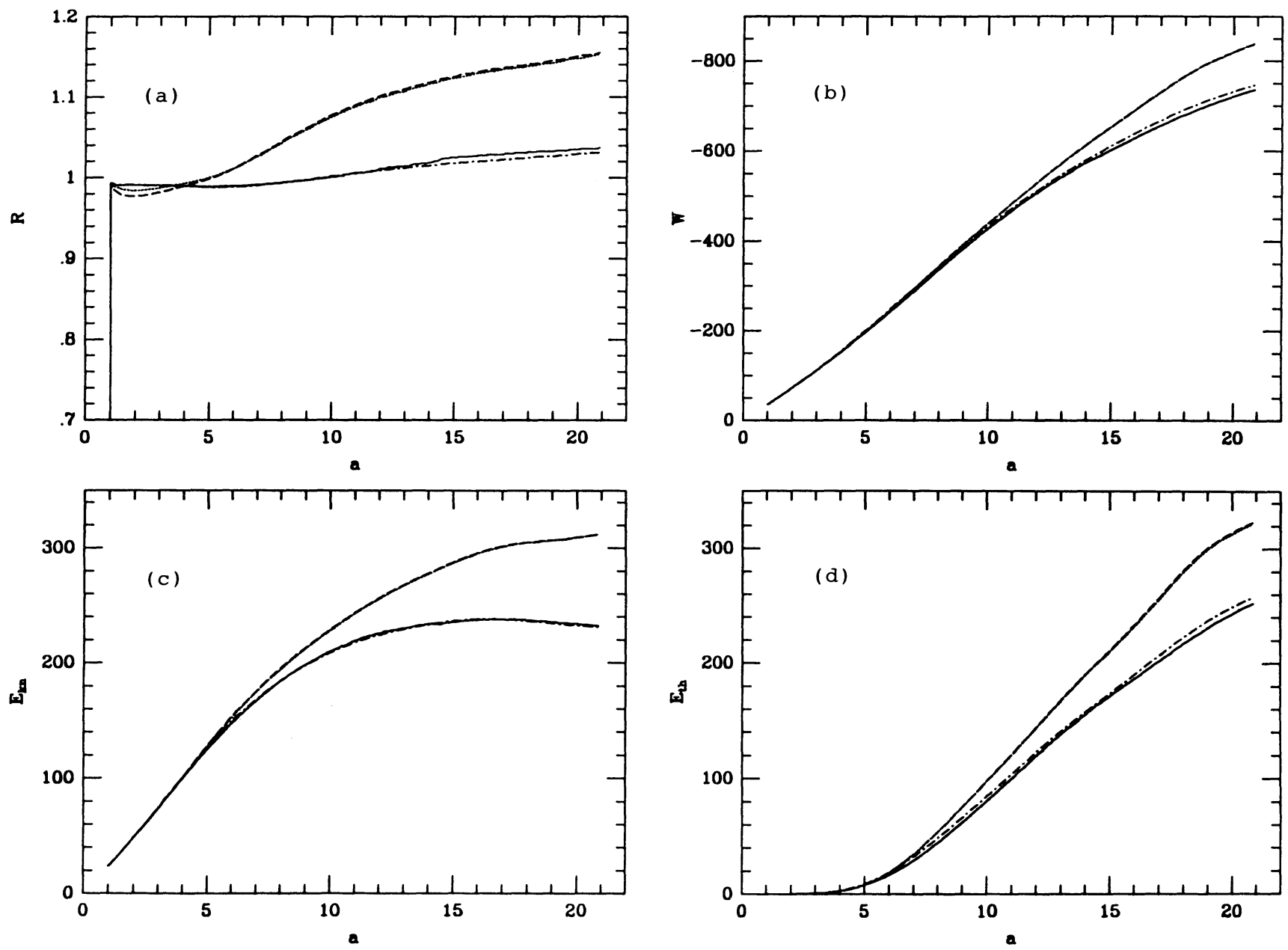

FIG. 11.-Conservation parameter, $R$ (see the text for the definition), gravitational energy, $W$, kinetic energy, $\mathscr{E}_{\text {kin }}$, and thermal energy, $\mathscr{E}_{\text {th }}$, as a function of the expansion parameter, $a$, for the three-dimensional simulations of a purely baryonic, adiabatic universe with the CDM initial power spectrum. The solid and the dashed lines are for TVD-ES code with and without the diffusive correction, respectively, while the dot-dashed and the dotted lines are for TVD-E code with and without the diffusive correction, respectively.

Here, we list the eigenvalues and the right and left eigenvectors of $\boldsymbol{A}_{x}(\boldsymbol{q})$ which is used to build the TVD scheme. The eigenvalues and eigenvectors of $\boldsymbol{A}_{\boldsymbol{y}}(\boldsymbol{q})$ and $\boldsymbol{A}_{z}(\boldsymbol{q})$ are obtained by properly permuting the indices. The eigvenvalues of $\boldsymbol{A}_{\boldsymbol{x}}(\boldsymbol{q})$ are

$$
\begin{gathered}
a_{1}=v_{x}-c, \\
a_{2}=v_{x}, \\
a_{3}=v_{x}, \\
a_{4}=v_{x}, \\
a_{5}=v_{x}+c,
\end{gathered}
$$

where the sound speed is $c=(\gamma p / \rho)^{1 / 2}$.

The corresponding right eigenvectors are

$$
\boldsymbol{R}_{1}=\left(\begin{array}{c}
1 \\
v_{x}-c \\
v_{y} \\
v_{z} \\
H-v_{x} c
\end{array}\right)
$$




$$
\begin{gathered}
\boldsymbol{R}_{2}=\left(\begin{array}{l}
0 \\
0 \\
1 \\
0 \\
v_{y}
\end{array}\right), \\
\boldsymbol{R}_{3}=\left(\begin{array}{c}
1 \\
v_{x} \\
v_{y} \\
v_{z} \\
\theta / 2
\end{array}\right), \\
\boldsymbol{R}_{4}=\left(\begin{array}{c}
0 \\
0 \\
0 \\
1 \\
v_{z}
\end{array}\right), \\
\boldsymbol{R}_{5}=\left(\begin{array}{c}
1 \\
v_{x}+c \\
v_{y} \\
v_{z} \\
+v_{x} c
\end{array}\right),
\end{gathered}
$$

where $H=(E+p) / \rho$ is the enthalpy and $\theta=v_{x}^{2}+v_{y}^{2}+v_{z}^{2}$.

The left eigenvectors which are orthonormal to the right eigenvector, $\boldsymbol{L}_{l} \cdot \boldsymbol{R}_{\boldsymbol{m}}=\delta_{l m}$, are

$$
\begin{gathered}
\boldsymbol{L}_{1}=\left[\frac{(\gamma-1) \theta / 2+c v_{x}}{2 c^{2}},-\frac{(\gamma-1) v_{x}+c}{2 c^{2}},-\frac{(\gamma-1) v_{y}}{2 c^{2}},-\frac{(\gamma-1) v_{z}}{2 c^{2}}, \frac{(\gamma-1)}{2 c^{2}}\right], \\
\boldsymbol{L}_{2}=\left(-v_{y}, 0,1,0,0\right), \\
\boldsymbol{L}_{3}=\left[1-\frac{(\gamma-1) \theta}{2 c^{2}}, \frac{(\gamma-1) v_{x}}{c^{2}}, \frac{(\gamma-1) v_{y}}{c^{2}}, \frac{(\gamma-1) v_{z}}{c^{2}},-\frac{\gamma-1}{c^{2}}\right], \\
\boldsymbol{L}_{5}=\left[\frac{\left(\gamma-v_{z}, 0,0,1,0\right),}{2 c^{2}},-\frac{(\gamma-1) v_{x}-c}{2 c^{2}},-\frac{(\gamma-1) v_{y}}{2 c^{2}},-\frac{(\gamma-1) v_{z}}{2 c^{2}}, \frac{(\gamma-1)}{2 c^{2}}\right] .
\end{gathered}
$$

In the TVD scheme which is based on the Eulerian grid, the flux is computed on the grid boundary while the physical quantities are defined in the grid center. We use the Roe approximate Riemann solution (Roe 1981) to get the averaged values of the physical quantities at the grid boundary:

$$
\begin{aligned}
& v_{x, i+1 / 2}=\frac{\sqrt{\rho_{i}} v_{x, i}+\sqrt{\rho_{i+1}} v_{x, i+1}}{\sqrt{\rho_{i}}+\sqrt{\rho_{i+1}}}, \\
& v_{y, i+1 / 2}=\frac{\sqrt{\rho_{i}} v_{y, i}+\sqrt{\rho_{i+1}} v_{y, i+1}}{\sqrt{\rho_{i}}+\sqrt{\rho_{i+1}}}, \\
& v_{z, i+1 / 2}=\frac{\sqrt{\rho_{i}} v_{z, i}+\sqrt{\rho_{i+1}} v_{z, i+1}}{\sqrt{\rho_{i}}+\sqrt{\rho_{i+1}}}, \\
& H_{i+1 / 2}=\frac{\sqrt{\rho_{i}} H_{i}+\sqrt{\rho_{i+1}} H_{i+1}}{\sqrt{\rho_{i}}+\sqrt{p_{i+1}}}, \\
& c_{i+1 / 2}=\left\{(\gamma-1)\left[H_{i+1 / 2}-\frac{1}{2}\left(v_{x, i+1 / 2}^{2}+v_{y, i+1 / 2}^{2}+v_{z, i+1 / 2}^{2}\right)\right]\right\}^{1 / 2} .
\end{aligned}
$$


APPENDIX B

\section{PROCEDURE TO BUILD THE THREE-DIMENSIONAL TVD CODE}

Here, we list briefly the procedure to build the three-dimensional TVD code with the eigenvalues and eigenvectors in Appendix A. The purpose of this appendix is to provide a short but complete description of steps needed to build the three-dimensional TVD code. For the details, e.g., why and how each step works, the choice of the values of the internal parameters, etc., refer the original reference (Harten 1983).

In updating the vector $\boldsymbol{q}^{n}$ to $q^{n+1}$, the fluxes along the $x, y$, and $z$ directions are included separated as if it is one-dimensional. The procedure to include the $x$-flux is following:

$$
\begin{aligned}
& L_{x} q_{i}^{n}=q_{i}^{n}-\frac{\Delta t^{n}}{\Delta x}\left(\bar{f}_{x, i+1 / 2}-\bar{f}_{x, i-1 / 2}\right) \\
& \bar{f}_{x, i+1 / 2}=\frac{1}{2}\left[F_{x}\left(q_{i}^{n}\right)+F_{x}\left(q_{i+1}^{n}\right)\right]-\frac{\Delta x}{2 \Delta t^{n}} \sum_{k=1}^{5} \beta_{k, i+1 / 2} R_{k, i+1 / 2}^{n}, \\
& \beta_{k, i+1 / 2}=Q_{k}\left(\frac{\Delta t^{n}}{\Delta x} a_{k, i+1 / 2}^{n}+\gamma_{k, i+1 / 2}\right) \alpha_{k, i+1 / 2}-\left(g_{k, i}+g_{k, i+1}\right), \\
& \alpha_{k, i+1 / 2}=L_{k, i+1 / 2}^{n} \cdot\left(q_{i+1}^{n}-q_{i-1}^{n}\right), \\
& \gamma_{k, i+1 / 2}=\left\{\begin{array}{ll}
\left(g_{k, i+1}-g_{k, i}\right) / \alpha_{k, i+1 / 2}, & \text { for } \alpha_{k, i+1 / 2} \neq 0 \\
0, & \text { for } \alpha_{k, i+1 / 2}=0
\end{array},\right. \\
& g_{k, i}=\operatorname{sign}\left(\tilde{g}_{k, i+1 / 2}\right) \max \left\{0, \min \left[\left|\tilde{g}_{k, i+1 / 2}\right|, \quad \tilde{g}_{k, i-1 / 2} \operatorname{sign}\left(\tilde{g}_{k, i+1 / 2}\right)\right]\right\}, \\
& \tilde{g}_{k, i+1 / 2}=\frac{1}{2}\left[Q_{k}\left(\frac{\Delta t^{n}}{\Delta x} a_{k, i+1 / 2}^{n}\right)-\left(\frac{\Delta t^{n}}{\Delta x} a_{k, i+1 / 2}^{n}\right)^{2}\right] \alpha_{k, i+1 / 2}, \\
& Q_{k}(\chi)=\left\{\begin{array}{ll}
{\left[\chi^{2} /(4 \epsilon)\right]+\epsilon,} & \text { for }|\chi|<2 \epsilon \\
|\chi|, & \text { for }|\chi| \geq 2 \epsilon,
\end{array} \quad \epsilon= \begin{cases}0.03, & \text { for } k=1 \text { and } 5 \\
0, & \text { for } k=2,3, \text { and } 4 .\end{cases} \right.
\end{aligned}
$$

Here, the time step $\Delta t^{n}$ is restricted by the usual Courant condition for the stability, $\Delta t^{n}<\Delta x /\left(\left|v_{x, i+1 / 2}^{n}\right|+c_{i+1 / 2}^{n}\right)$. The same procedure is applied for $y$ - and $z$-fluxes. Then, the updated vector is

$$
q^{n+1}=L_{z} L_{y} L_{x} q^{n}
$$

In order to get a second-order accuracy, the order to include the fluxes is permuted by the Strang-type dimensional splitting (Strang 1968) as the following way: $L_{z} L_{y} L_{x}, L_{x} L_{y} L_{z}, L_{x} L_{z} L_{y}, L_{y} L_{z} L_{x}, L_{y} L_{x} L_{z}$, and then $L_{z} L_{x} L_{y}$.

As mentioned in $\S 4.1$, the TVD code described in the above spreads the contact discontinuity over six to seven cells. The contact discontinuity can be steepened to to be spread over two to three cells by adding the steepening scheme in which $g_{k, i}$ is replaced by $g_{k, i}+\theta_{k, i} \bar{g}_{k, i}$ for $k=3$ :

$$
\begin{gathered}
\theta_{k, i}=\left\{\begin{array}{ll}
\left|\alpha_{k, i+1 / 2}-\alpha_{k, i-1 / 2}\right| /\left(\left|\alpha_{k, i+1 / 2}\right|+\left|\alpha_{k, i-1 / 2}\right|\right), & \text { for }\left(\left|\alpha_{k, i+1 / 2}\right|+\left|\alpha_{k, i-1 / 2}\right|\right) \neq 0 \\
0, & \text { for }\left(\left|\alpha_{k, i+1 / 2}\right|+\left|\alpha_{k, i-1 / 2}\right|\right)=0
\end{array},\right. \\
\bar{g}_{k, i}=\operatorname{sign}\left(\alpha_{k, i+1 / 2}\right) \max \left\{0, \min \left[\operatorname{sign}\left(\alpha_{k, i+1 / 2}\right) \sigma_{k, i-1 / 2} \alpha_{k, i-1 / 2}, \sigma_{k, i+1 / 2}\left|\alpha_{k, i+1 / 2}\right|\right]\right\}, \\
\sigma_{k, i+1 / 2}=\frac{1}{2}\left[1-Q_{k}\left(\frac{\Delta t^{n}}{\Delta x} a_{k, i+1 / 2}\right)\right] .
\end{gathered}
$$

\section{APPENDIX C}

\section{EIGENVALUES AND EIGENVECTORS FOR THE MODIFIED HYDRODYNAMIC EQUATIONS}

The mass and momentum conservation equations in (2.1) and (2.2) with the modified entropy equation which we use for the flow in the unshocked regions are written in vector form as

$$
\begin{gathered}
\partial_{t} \boldsymbol{q}+\partial_{x} \boldsymbol{F}_{x}+\partial_{y} \boldsymbol{F}_{y}+\partial_{z} \boldsymbol{F}_{z}=0, \\
\boldsymbol{q}=\left(\begin{array}{c}
\rho \\
\rho v_{x} \\
\rho v_{y} \\
\rho v_{z} \\
S
\end{array}\right), \quad \boldsymbol{F}_{x}=\left(\begin{array}{c}
\rho v_{x} \\
\rho v_{x}^{2}+S \rho^{\gamma-1} \\
\rho v_{x} v_{y} \\
\rho v_{x} v_{z} \\
S v_{x}
\end{array}\right), \quad \boldsymbol{F}_{y}=\left(\begin{array}{c}
\rho v_{x} v_{y} \\
\rho v_{y}^{2}+S \rho^{\gamma-1} \\
\rho v_{y} v_{z} \\
S v_{y}
\end{array}\right), \quad \boldsymbol{F}_{z}=\left(\begin{array}{c}
\rho v_{z} \\
\sigma v_{x} v_{z} \\
\rho v_{y} v_{z} \\
\rho v_{z}^{2}+S \rho^{\gamma-1} \\
S v_{z}
\end{array}\right),
\end{gathered}
$$

where the equation of state is $S=p / \rho^{\gamma-1}$.

(C) American Astronomical Society - Provided by the NASA Astrophysics Data System 
The above set of equations also form a hyperbolic system. It has the same eigenvalues as the set of the hydrodynamic equations in equations (A3)-(A5), as expected. The corresponding right eigenvectors are

$$
\begin{aligned}
& \boldsymbol{R}_{1}=\left(\begin{array}{c}
1 \\
v_{x}-c \\
v_{y} \\
v_{z} \\
S / \rho
\end{array}\right), \\
& \boldsymbol{R}_{2}=\left(\begin{array}{l}
0 \\
0 \\
1 \\
0 \\
0
\end{array}\right), \\
& \boldsymbol{R}_{3}=\left(\begin{array}{c}
1 \\
v_{x} \\
v_{y} \\
v_{z} \\
-(\gamma-1) S / \rho
\end{array}\right) \text {, } \\
& \boldsymbol{R}_{4}=\left(\begin{array}{l}
0 \\
0 \\
0 \\
1 \\
0
\end{array}\right), \\
& \boldsymbol{R}_{5}=\left(\begin{array}{c}
1 \\
v_{x}+c \\
v_{y} \\
v_{z} \\
S / \rho
\end{array}\right) \text {. }
\end{aligned}
$$

The left eigenvectors are

$$
\begin{gathered}
\boldsymbol{L}_{1}=\left(\frac{\gamma-1}{2 \gamma}+\frac{v_{x}}{2 c},-\frac{1}{2 c^{2}}, 0,0, \frac{\rho}{2 \gamma S}\right), \\
\boldsymbol{L}_{2}=\left(-v_{y}, 0,1,0,0\right), \\
\boldsymbol{L}_{3}=\left(\frac{1}{\gamma}, 0,0,0,-\frac{\rho}{\gamma S}\right), \\
\boldsymbol{L}_{4}=\left(-v_{z}, 0,0,1,0\right), \\
\boldsymbol{L}_{5}=\left(\frac{\gamma-1}{2 \gamma}-\frac{v_{x}}{2 c}, \frac{1}{2 c^{2}}, 0,0, \frac{\rho}{2 \gamma S}\right) .
\end{gathered}
$$

With the modified entropy equation instead of the total energy equation, the simple Roe-type Riemann solution cannot be constructed. Hence, we used the following averaging scheme to get the values of the physical quantities at the grid boundary:

$$
\begin{gathered}
\rho_{i+1 / 2}=\frac{\rho_{i}+\rho_{i+1}}{2}, \\
v_{x, i+1 / 2}=\frac{\sqrt{\rho_{i}} v_{x, i}+\sqrt{\rho_{i+1}} v_{x, i+1}}{\sqrt{\rho_{i}}+\sqrt{\rho_{i+1}}}, \\
v_{y, i+1 / 2}=\frac{\sqrt{\rho_{i}} v_{y, i}+\sqrt{\rho_{i+1}} v_{y, i+1}}{\sqrt{\rho_{i}}+\sqrt{\rho_{i+1}}},
\end{gathered}
$$




$$
\begin{gathered}
v_{z, i+1 / 2}=\frac{\sqrt{\rho_{i}} v_{z, i}+\sqrt{\rho_{i+1}} v_{z, i+1}}{\sqrt{\rho_{i}}+\sqrt{\rho_{i+1}}}, \\
S_{i+1 / 2}=\frac{\sqrt{\rho_{i}} S_{i}+\sqrt{\rho_{i+1}} S_{i+1}}{\sqrt{\rho_{i}}+\sqrt{\rho_{i+1}}}, \\
c_{i+1 / 2}=\gamma S_{i+1 / 2} \rho_{i+1 / 2}^{\gamma-2} .
\end{gathered}
$$

As pointed in Harten (1983), the quality of the TVD code does not sensitively depend on the averaging scheme.

\section{APPENDIX D}

\section{PROCEDURE TO BUILD THE COSMOLOGICAL TVD CODE}

Here, we list schematically the procedure to build our cosmological hydrodynamic code. We has used the TVD-ES code to update the hydrodynamics of the baryonic matter. And we have adopted the Lax-Wendroff scheme for the time integration of the dark matter, instead of the common leapfrog scheme, since the leapfrog scheme requires a constant time step.

Step 0.- We have $\rho_{\mathrm{BM}}^{n}, v_{i, \mathrm{BM}}^{n}$, and $p^{n}$ for the baryonic matter, $x_{i}^{n}$ and $v_{i, \mathrm{DM}}^{n}$ for the dark matter, and $\phi^{n}$ at $t^{n}$ (or $a^{n}$ ).

Step 1.-Preprocessor for the baryonic matter to get $\tilde{v}_{i, \mathrm{BM}}^{n}$ and $\tilde{p}^{n}$,

$$
\begin{gathered}
\tilde{v}_{i, \mathrm{BM}}^{n}=v_{i, \mathrm{BM}}^{n}-\frac{\Delta t^{n}}{2} \frac{\dot{a}^{n}}{a^{n}} v_{i, \mathrm{BM}}^{n}-\frac{\Delta t^{n}}{2} \frac{1}{a^{n}} \frac{\partial \phi^{n}}{\partial x_{i}}, \\
\tilde{p}^{n}=p_{n}-\frac{\Delta t^{n}}{2} \frac{2 \dot{a}^{n}}{a^{n}} p^{n} .
\end{gathered}
$$

Step 2.- Hydrodynamic step for the baryonic matter, call TVD-ES with $\rho_{\mathrm{BM}}^{n}, \tilde{v}_{i, \mathrm{BM}}^{n}$, and $\tilde{p}^{n}$

$$
\begin{gathered}
\rho_{\mathrm{BM}}^{n+1}=\rho_{\mathrm{BM}}^{n}+\Delta \rho_{\mathrm{BM}}^{\mathrm{TVD}}\left(\rho_{\mathrm{BM}}^{n}, \tilde{v}_{i, \mathrm{BM}}^{n}, \tilde{p}^{n}\right), \\
\left(v_{i, \mathrm{BM}}^{n+1}\right)_{\mathrm{hydro}}=v_{i, \mathrm{BM}}^{n}+\Delta v_{i, \mathrm{BM}}^{\mathrm{TVD}}\left(\rho_{\mathrm{BM}}^{n}, \tilde{v}_{i, \mathrm{BM}}^{n}, \tilde{p}^{n}\right), \\
\left(p^{n+1}\right)_{\mathrm{hydro}}=p^{n}+\Delta p^{\mathrm{TVD}}\left(\rho_{\mathrm{BM}}^{n}, \tilde{v}_{i, \mathrm{BM}}^{n}, \tilde{p}^{n}\right) .
\end{gathered}
$$

Step 3.- $\left(n+\frac{1}{2}\right)$ step for the dark matter,

$$
\begin{gathered}
x_{i}^{n+1 / 2}=x_{i}^{n}+\frac{\Delta t^{n}}{2} \frac{v_{i, \mathrm{DM}}^{n}}{a^{n}}, \\
v_{i, \mathrm{DM}}^{n+1 / 2}=v_{i, \mathrm{DM}}^{n}-\frac{\Delta t^{n}}{2} \frac{\dot{a}^{n}}{a^{n}} v_{i, \mathrm{DM}}^{n}-\frac{\Delta t^{n}}{2} \frac{1}{a^{n}} \frac{\partial \phi^{n}}{\partial x_{i}} .
\end{gathered}
$$

Step 4.-Potential at $\left(n+\frac{1}{2}\right)$,

$$
\rho_{\mathrm{tot}}^{n+1 / 2}=\frac{1}{2}\left(\rho_{\mathrm{BM}}^{n}+\rho_{\mathrm{BM}}^{n+1}\right)+\rho_{\mathrm{DM}}^{n+1 / 2} \Rightarrow \phi^{n+1 / 2} .
$$

Step 5.-Gravitational step for the baryonic matter,

$$
\left(v_{i, \mathrm{BM}}^{n+1}\right)_{\mathrm{hydro}+\mathrm{grav}}=\left(v_{i, \mathrm{BM}}^{n+1}\right)_{\mathrm{hydro}}-\Delta t^{n} \frac{1}{a^{n+1 / 2}} \frac{\partial \phi^{n+1 / 2}}{\partial x_{i}} .
$$

Step 6.-Expansion step for the baryonic matter,

$$
\begin{gathered}
v_{i, \mathrm{BM}}^{n+1}=\frac{\left(v_{i, \mathrm{BM}}^{n+1}\right)_{\mathrm{hydro}+\mathrm{grav}}-\left(\Delta t^{n} / 2\right)\left(\dot{a}^{n+1 / 2} / a^{n+1 / 2}\right) v_{i, \mathrm{BM}}^{n}}{1+\left(\Delta t^{n} / 2\right)\left(\dot{a}^{n+1 / 2} / a^{n+1 / 2}\right)}, \\
p^{n+1}=\frac{\left(p^{n+1}\right)_{\mathrm{hydro}}-\Delta t^{n}\left(\dot{a}^{n+1 / 2} / a^{n+1 / 2}\right) p^{n}}{1+\Delta t^{n}\left(\dot{a}^{n+1 / 2} / a^{n+1 / 2}\right)} .
\end{gathered}
$$

Step 7.- $(n+1)$ step for the dark matter,

$$
\begin{gathered}
x_{i}^{n+1}=x_{i}^{n}+\Delta t^{n}\left(v_{i, \mathrm{DM}}^{n+1 / 2} / a^{n+1 / 2}\right), \\
v_{i, \mathrm{DM}}^{n+1}=v_{i, \mathrm{DM}}^{n}-\Delta t^{n}\left(\dot{a}^{n+1 / 2} / a^{n+1 / 2}\right) v_{i, \mathrm{DM}}^{n+1 / 2}-\Delta t^{n}\left(1 / a^{n+1 / 2}\right)\left(\partial \phi^{n+1 / 2} / \partial x_{i}\right) .
\end{gathered}
$$

Step 8.-Potential at $(n+1)$,

$$
\rho_{\mathrm{tot}}^{n+1}=\rho_{\mathrm{BM}}^{n+1}+\rho_{\mathrm{DM}}^{n+1} \Rightarrow \phi^{n+1}
$$


Step 9.- -Now, we have $\rho_{\mathrm{BM}}^{n+1}, v_{i, \mathrm{BM}}^{n+1}$, and $p^{n+1}$ for the baryonic matter, $x_{i}^{n+1}$ and $v_{i, \mathrm{DM}}^{n+1}$ for the dark matter, and $\phi^{n+1}$ at $t^{n+1}$ (or $\left.a^{n+1}\right)$.

I Here, the dark matter density is calculated from the dark matter particles with the cloud-in-cell mass assignment scheme. The gravitational potential is calculated with the fast Fourier transformation technique. The gravitational force on the baryonic grid is calculated by finite differencing the potential fields at two adjacent grids and the gravitational force on each dark matter particle is calculated by the linear interpolation of the forces at the eight neighboring grids using the cloud-in-cell scheme.

\section{REFERENCES}

Bertschinger, E., \& Gelb, J. 1991, Comput. Phys., 5, 164

Cen, R. 1992, ApJS, 78, 341

Cen, R., Jameson, A., Liu, F., \& Ostriker, J. P. 1990, ApJ, 362, L41

Cen, R., \& Ostriker, J. P. 1992a, ApJ, 393, 22

1992b, ApJ, 399, 331

1992c, ApJ, 399, L113

Cen, R., Ostriker, J. P., Spergel, D. N., \& Turok, N. 1991, ApJ, 383,

Centrella, J. M., Gallagher, J. S., Melott, A. L., \& Bushouse, H. A. 1988, ApJ, 333,24

Chiang, W. H., Ryu, D., \& Vishniac, E. T. 1989, ApJ, 339, 603

Colella, P., \& Woodward, P. R. 1984, J. Comp. Phys., 54, 174

Efstathiou, G., Davis, M., Frenk, C. S., \& White, S. D. M. 1985, ApJS, 57, 241

Giacconi, R., Gursky, H., Paolini, F., \& Rossi, B. 1962, Phys. Rev. Lett., 9, 439

Harten, A. 1983, J. Comp. Phys., 49, 357

Katz, N., Hernquist, L., \& Weinberg, D. H. 1992, preprint

Kurki, Suonio, H., Matzner, R. A., Olive, K. A., \& Schramm, D. N. 1990, ApJ, 353, 406
Melott, A. L. 1986, Phys. Rev. Lett., 56, 1992

Ostriker, J. P., \& Vishniac, E. T. 1986, ApJ, 306, L51

Park, C. 1990, MNRAS, 242, 59P

1991, Ph.D. thesis, Princeton Univ.

Peebles, P. J. E. 1980, The Large-Scale Structure of the Universe (Princeton: Princeton Univ. Press) 1986, Nature, 32, 1

Roe, P. L. 1981, J. Comp. Phys., 43, 357

Ryu, D., Vishniac, E. T., \& Chiang, W. H. 1990, ApJ, 354, 389

Strang, G. 1968, SIAM J. Num. Anal., 5, 506

Walker, T., Steigman, G., Schramm, D. N., Olive, K. A., \& Kang, H. S. 1991, ApJ, 376, 51

Weinberg, D. H., \& Gunn, J. E. 1990, MNRAS, 247, 260

Yuan, W., Centrella, J. M., \& Norman, M. L. 1991, ApJ, 376, L29

Zel'dovich, Ya. B., \& Sunyaev, R. A. 1969, A\&A, 20, 189 